\title{
Human airway-like multilayered tissue on 3D-TIPS printed thermoresponsive elastomer/collagen hybrid scaffolds
}

DOI:

10.1016/j.actbio.2020.07.013

\section{Document Version}

Accepted author manuscript

Link to publication record in Manchester Research Explorer

\section{Citation for published version (APA):}

Wu, L., Magaz, A., Huo, S., Darbyshire, A., Loizidou, M., Emberton, M., Birchall, M., \& Song, W. (2020). Human airway-like multilayered tissue on 3D-TIPS printed thermoresponsive elastomer/collagen hybrid scaffolds. Acta Biomaterialia. https://doi.org/10.1016/j.actbio.2020.07.013

\section{Published in:}

Acta Biomaterialia

\section{Citing this paper}

Please note that where the full-text provided on Manchester Research Explorer is the Author Accepted Manuscript or Proof version this may differ from the final Published version. If citing, it is advised that you check and use the publisher's definitive version.

\section{General rights}

Copyright and moral rights for the publications made accessible in the Research Explorer are retained by the authors and/or other copyright owners and it is a condition of accessing publications that users recognise and abide by the legal requirements associated with these rights.

\section{Takedown policy}

If you believe that this document breaches copyright please refer to the University of Manchester's Takedown Procedures [http://man.ac.uk/04Y6Bo] or contact uml.scholarlycommunications@manchester.ac.uk providing relevant details, so we can investigate your claim.

\section{OPEN ACCESS}




\section{Journal Pre-proof}

Human airway-like multilayered tissue on 3D-TIPS printed thermoresponsive elastomer/collagen hybrid scaffolds

Linxiao Wu , Adrián Magaz, Suguo Huo, Arnold Darbyshire, Marilena Loizidou, Mark Emberton, Martin Birchall, Wenhui Song

PII:

DOI:

Reference:

To appear in:

Received date:

Revised date:

Accepted date:
S1742-7061(20)30398-6

https://doi.org/10.1016/j.actbio.2020.07.013

ACTBIO 6821

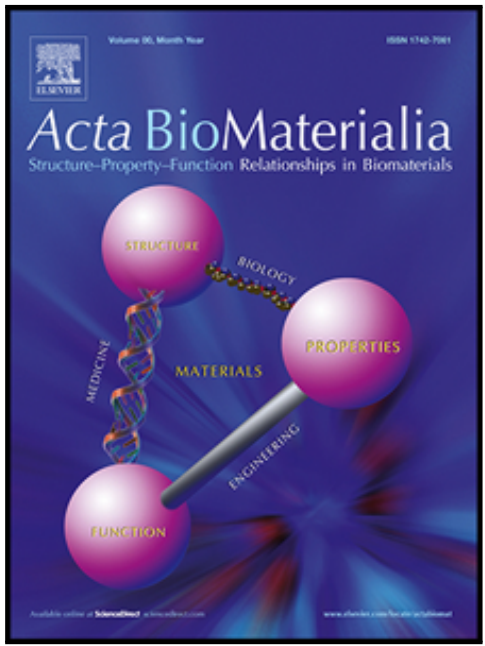

Please cite this article as: Linxiao Wu, Adrián Magaz, Suguo Huo, Arnold Darbyshire, Marilena Loizidou, Mark Emberton, Martin Birchall, Wenhui Song, Human airway-like multilayered tissue on 3D-TIPS printed thermoresponsive elastomer/collagen hybrid scaffolds, Acta Biomaterialia (2020), doi: https://doi.org/10.1016/j.actbio.2020.07.013

This is a PDF file of an article that has undergone enhancements after acceptance, such as the addition of a cover page and metadata, and formatting for readability, but it is not yet the definitive version of record. This version will undergo additional copyediting, typesetting and review before it is published in its final form, but we are providing this version to give early visibility of the article. Please note that, during the production process, errors may be discovered which could affect the content, and all legal disclaimers that apply to the journal pertain.

(C) 2020 Acta Materialia Inc. Published by Elsevier Ltd. All rights reserved. 


\section{Human airway-like multilayered tissue on 3D-TIPS printed thermoresponsive elastomer/collagen hybrid scaffolds}

Linxiao $\mathrm{Wu}^{\mathrm{a}, \mathrm{b}}$, Adrián Magaz ${ }^{\mathrm{a}, 1}$, Suguo Huo ${ }^{c}$, Arnold Darbyshire ${ }^{\mathrm{a}}$, Marilena Loizidou ${ }^{\mathrm{a}}$, Mark Emberton ${ }^{\mathrm{a}}$, Martin Birchall ${ }^{\mathrm{d}}$, Wenhui Song ${ }^{\mathrm{a}, *}$

${ }^{a}$ Centre for Biomaterials in Surgical Reconstruction and Regeneration, Division of Surgery \& Interventional Science, University College London, NW3 2PF, London, United Kingdom

${ }^{\mathrm{b}}$ Medical College, China Three Gorges University, Yichang 443002, P.R. China

'London Centre for Nanotechnology (LCN), 17-19 Gordon Street, WC1H OAH, London, United Kingdom dUCL Ear Institute, Royal National Throat, Nose and Ear Hospital, University College London, 330 Gray's Inn Road, WC1X 8DA, London, United Kingdom

*Corresponding author, e-mail: w.song@ucl.ac.uk

${ }^{1}$ Current address: Department of Materials and Henry Royce Institute, The University of Manchester, Sackville Street, M13 9PL, Manchester, United Kingdom 


\section{Graphical abstract}

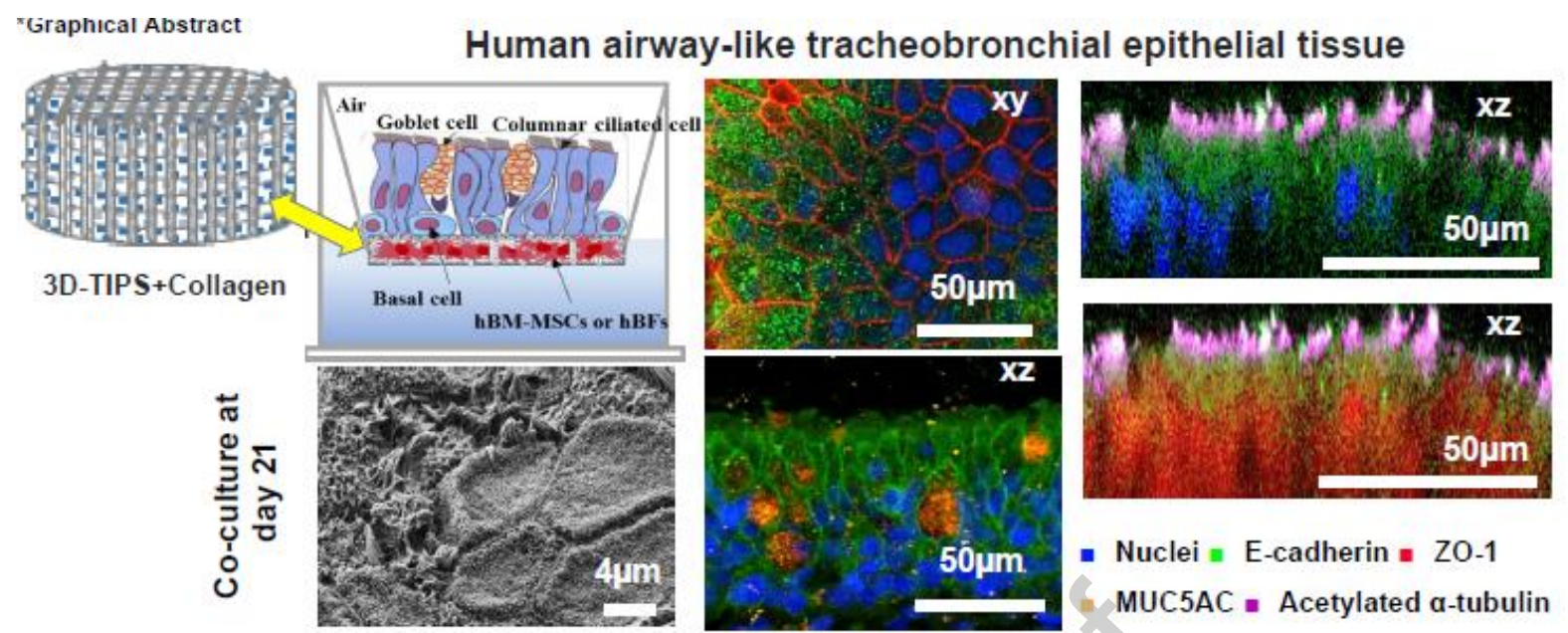




\section{Abstract}

Developing a biologically representative complex tissue of the respiratory airway is challenging, however, beneficial for treatment of respiratory diseases, a common medical condition representing a leading cause of death in the world. This study reports a successful development of synthetic human tracheobronchial epithelium based on interpenetrated hierarchical networks composed of a reversely 3D printed porous structure of a thermoresponsive stiffness-softening elastomer nanohybrid impregnated with collagen nanofibrous hydrogel in vitro. Human bronchial epithelial cells (hBEpiCs) were able to attach and grow into an epithelial monolayer on the hybrid scaffolds co-cultured with either human bronchial fibroblasts (hBFs) or human bone-marrow derived mesenchymal stem cells (hBM-MSCs), with substantial enhancement of mucin expression, ciliation, well-constructed intercellular tight junctions and adherens junctions. The multi-layered co-culture 3D scaffolds consisting of a top monolayer of differentiated epithelium, with either hBFs or hBM-MISCs proliferating within the hyperelastic nanohybrid scaffold underneath, created a tissue analogue of the upper respiratory tract, validating these 3D printed guided scaffolds as a platform to support co-culture and cellular organization. In particular, hBM-MSCs in the co-culture system promoted an overall matured physiological tissue analogue of the respiratory system, a promising synthetic tissue for drug discovery, tracheal repair and reconstruction.

\section{Keywords}

Nanohybrid elastomer, 3D-TIPS, 3D printing, hybrid interpenetrating network, respiratory epithelium, co-culture 


\section{Statement of significance}

Respiratory diseases are a common medical condition and represent a leading cause of death in the world. However, the epithelium is one of the most challenging tissues to culture in vitro, and suitable tracheobronchial models, physiologically representative of the innate airway, remain largely elusive. This study presents, for the first time, a systematic approach for the development of functional multilayered epithelial synthetic tissue in vitro via co-culture on a 3D-printed thermoresponsive elastomer interpenetrated with a collagen hydrogel network. The viscoelastic nature of the scaffold with stiffness softening at body temperature provide a promising matrix for soft tissue engineering. The results presented here provide new insights about the epithelium at different surfaces and interfaces of co-culture, and pave the way to offer a customizable reproducible technology to generate physiologically relevant 3D biomimetic systems to advance our understanding of airway tissue regeneration. 


\section{Introduction}

Respiratory diseases are a common medical condition and represent a leading cause of death in the world [1]. For instance, cystic fibrosis and chronic pulmonary disease alone have been identified as the fifth cause of death and they are predicted to rise in the coming years [2]. For that reason alone, gaining an understanding of the mechanisms of the airway injury and repair process is a main focus in the field of respiratory medicine [3]. In particular, the airway epithelium is of interest since it acts as a primary protective barrier to the external environment defending against toxins, pollutants and pathogens, and maintains homeostasis by regulating the innate immune response [4-6]. There is also a growing need to develop synthetic respiratory tissues/organs-physiologically representative of the innate airway-for surgical reconstruction and upcoming therapeutics whose primary route of delivery is through the airway tract, or to study tissue disease $[7,8]$. However, despite numerous attempts made by several groups, the creation of a multilayer-structured airway with physiological functions has to date remained elusive [9].

The reasons relate, in part, to the many integrated functions that the tracheobronchial epithelium above the basement membrane is required to fulfill. These functions comprise: barrier integrity, mucus production, antimicrobial capability, and cilia motility [10]. Three main cell types-ciliated, secretory (primarily mucus-secreting goblet cells) and basal cells-support these functions. These cell types need to be re-capitulated in any synthetic construct and be arranged in a manner that permits the functions to be fulfilled. Three main factors as well have been identified as necessary for development towards airway tract replacements [10]: these are a basal lamina equivalent with collagen fibers for cell-cell interaction and polarization, mesenchymal fibroblasts, and the presence of an air-liquid interface system for the proliferation and differentiation of bronchial epithelial cells [11].

Creating a scaffold that will permit cells to fulfill their function and maintain anatomical patency remains a significant challenge. The two main options that have been tried come with inherent challenges. Decellularized lung/trachea scaffolds have failed to confer sufficient structural integrity due to compromised cartilage and tend to collapse, therefore a stiffer stent is often required to provide additional mechanical support to the weak graft during implantation, which could cause extrusion failure $[9,12-15]$. Despite promising epithelium, there is also little evidence of new cartilage formation within the dense decellularized matrix that prevents cell penetration and proliferation $[15,16]$. Scaffolds constructed from synthetic materials, conversely, have remained ridged but failed to restore blood 
supply or too rigid to integrate with the cells/tissue [17]. The challenges for developing both structural and functional scaffolds still remain: air-sealing capabilities, the delay in vascularization leading to necrosis, re-epithelialization or mechanical mismatch are the major problems $[9,18]$.

Alternatives abound but few long-term successes have been achieved. Tani et al. developed a fibrous trachea-bronchial tissue architecture based on porous collagen scaffolds [19,20], but the barrier function was not properly maintained at the cell-material surface interface due to excessive permeability. O'Leary et al., had more success by using a collagen-hyaluronan bi-layered scaffold that facilitated the culture of bronchial epithelial cells on a 2D layer and their co-culture with lung fibroblasts [21], but without addressing the critical issue of the weak mechanical properties of the biopolymer scaffolds.

3D printing technologies [22] offer precise spatial control with high resolution, uniformity in the interconnectivity of pores, high accuracy and great geometry complexity, with the benefit of being highly reproducible. Fused deposition modelling and micro-extrusion-based 3D printing are two widely used techniques for the manufacture of polymer/hydrogel-based scaffolds. However, limitations persist. The choice of printable materials is limited and printing soft materials or solution with desirable cell attachment and viability remains challenging. The resolution to which they can be printed is, as yet, not optimal [23]. One way through to overcome these issues is to 3D print in an indirect manner, such as 3D printing sacrificial molds by incorporating thermally induced phase-separation (3D-TIPS) [24]. Uniform phase-separation and phase transition of the polymer solution at a large interface of network within the printed sacrificial preform can lead to the creation of full-scale scaffolds with bespoke anatomical complex geometry, hierarchically interconnected pores, controlled porosity and self-assembled nanophase structure.

A wide hierarchy of porous soft elastomer scaffolds with tunable stiffness were fabricated by 3D-TIPS using a non-degradable solution-based poly(urea-urethane) terminated by polyhedral oligomeric silsesquioxane (PUU-POSS) [24], achieved by dual control of the 3D printing infill density and phase separation of the polymer solution at different temperatures. Thermoresponsive dynamic stiffness softening was observed when the scaffolds were subjected to body temperature near the phase transition, i.e. the melting point of the crystalline domains of the soft segments of PUU. Systematic studies have proven the different roles of the hierarchical porosity and stiffness softening of these scaffolds in modulating cell growth and differentiation in vitro [24-26], tissue ingrowth, vascularization and macrophage polarization in vivo [27]. In particular, the initial stiffness and subsequent stiffness softening of these scaffolds at body temperature were found to promote cellular proliferation of both 
human dermal fibroblasts (hDFs) and human bone-marrow derived mesenchymal stem cells (hBMMSCs), and regulate as well their differentiation towards the chondrogenic and osteogenic lineages $[24,25]$. On the other hand, the hierarchical porous structure and resulting large surface area of the scaffold were believed to enhance protein adsorption, cellular attachment and nutrient/gas exchange flow during cell culture. The hBM-MSCs seeded 3D-TIPS constructs after an extended differentiation post-stiffness softening were proven to possess cartilage-like compression mechanical properties [25], which would be suitable for further development of tracheal reconstruction.

This paper reports the creation of a biofunctionalized bespoke 3D-TIPS printed elastomer-scaffold coated with collagen hydrogel that permits the culture of bronchial epithelium on monoculture and coculture conditions with human bronchial fibroblasts (hBFs) or hBM-MSCs (Scheme 1). The hybrid scaffold developed here is composed of interpenetrated porous networks of a soft poly(urea-urethane) nanohybrid impregnated with collagen hydrogel network. These scaffolds were incorporated as the sublayer of an epithelial in vitro model co-cultured with either hBFs or hBM-MSCs. The feasibility of the hybrid scaffolds to support growth and differentiation of bronchial epithelial cells and its ability to support the co-culture with either hBFs or hBM-MSCs have been testified. The formation of an epithelial barrier, the structure and biofunctions of columnar ciliated epithelial and mucus-secreting goblet cells at the air-liquid interface of the synthetic tracheal tissue have been systematically characterized.

\section{Materials and Methods}

\subsection{Scaffold fabrication}

Nanohybrid elastomer scaffolds based on poly(urea-urethane) (PUU) terminated by polyhedral oligomeric silsesquioxane (POSS) were manufactured by a 3D printed thermally induced phaseseparation (3D-TIPS) process following a previously described protocol [24]. Briefly, a PUU-POSS elastomer solution was synthesized and injected into 3D printed poly(vinyl alcohol) (PVA) preforms (100 $\mathrm{mm} \times 100 \mathrm{~mm} \times 1.2 \mathrm{~mm}$ ) with an orthogonal $50 \%$ infill density, used as water soluble negative sacrificial molds. PUU-POSS was then frozen within the preforms at $-20^{\circ} \mathrm{C}$ for $24 \mathrm{~h}$, followed by coagulation in an iced water bath for another $24 \mathrm{~h}$ and further washed by deionized water (Scheme 1A). PUU-POSS discs (16 $\mathrm{mm}$ diameter, $1 \mathrm{~mm}$ thickness) were then cut from the PUU-POSS scaffolds.

Collagen hydrogel functionalized 3D-TIPS scaffolds (3D-TIPS+Collagen) were fabricated by infusing a collagen solution $(2 \mathrm{mg} / \mathrm{mL}$ ) into the scaffold (16 mm diameter, $1 \mathrm{~mm}$ thickness) and subsequently compressed by using a process of Real Architecture for 3D Tissues (RAFT) (Scheme 1A) [28]. Briefly, 84\% $(\mathrm{v} / \mathrm{v})$ rat tail collagen type I solution (First Link, UK) was mixed with $10 \%(\mathrm{v} / \mathrm{v}) 10 \times$ Eagle's minimal 
essential medium (MEM) (Thermo Fisher, UK) until a homogenous yellow solution was obtained. Then, a $6 \%(\mathrm{v} / \mathrm{v}) \mathrm{NaOH} / \mathrm{HEPES}$ neutralizing solution (10M NaOH and 1M HEPES in a volume ratio of 0.198:1) was added and gently mixed. The mixture was held on ice prior to placing $1 \mathrm{~mL}$ of solution per scaffold on a 24-well plate. This was incubated for $15 \mathrm{~min}$ at $37^{\circ} \mathrm{C}$ to allow for collagen gelatinization prior to gentle wicking of water using hydrophilic porous absorbers (RAFT 3D systems; Lonza, UK), placed on top for 15 min at room temperature for liquid removal and pressed into the 3D-TIPS scaffold (Scheme 1A).

Following absorption, the absorbers were removed, resulting in about $1.68 \mathrm{mg}$ of collagen hydrogel per scaffold (taken into account the dilution factor of the collagen solution and the porosity of the scaffold (96.89\%)) with collagen concentration $8.7 \mathrm{mg} / \mathrm{mL}$.

\subsection{Physico-mechanical characterization}

The top surface of dried scaffolds (i.e. 3D-TIPS and 3D-TIPS+Collagen) was examined under a field emission scanning electron microscope (Zeiss Supra 35VP FE-SEM, Germany). A JEOL2100 FEG-TEM (Japan) was used to examine the nanophase structure of the scaffolds. The TEM samples were embedded in an aromatic acrylic resin (LR White Embedding Medium, EMS), cryo-microtomed into a thin section using a CR-X cryosectioning system (RMC Boeckeler), and then stained by Ruthenium tetroxide $\left(\mathrm{RuO}_{4}\right)$ vapour.

Samples ( $n=6$ per scaffold type) at wet condition were tested at room temperature in tensile and compression mode, prior to and after incubation at $37^{\circ} \mathrm{C}$ for 28 days. An Instron 5655 tester (Instron Ltd.; Norwood, USA) with a $5 \mathrm{~mm} / \mathrm{min}$ rate and a $500 \mathrm{~N}$ cell load was used to subject the samples to static tensile and compression tests. Dynamic mechanical properties were also tested in tensile and compression mode in a bioreactor at $37^{\circ} \mathrm{C}$ in water using an ElectroForce Biodynamic ${ }^{\circ}$ Test Instrument 5160 (TA, USA) with a $200 \mathrm{~N}$ load cell. Samples were loaded with a sinusoidal ramp of constant frequency of $1 \mathrm{~Hz}$ with a controlled strain (tensile strain or compression strain) of $25 \%$ at increasing cycles, up to 200,200 cycles per sample.

\subsection{In vitro testing}

\subsubsection{Cell selection and culture media}

Human bronchial epithelial cells (hBEpiCs) (ScienCell, California, USA) were used for monoculture and co-culture experiments. HBEpiCs were isolated from human bronchi, cryopreserved at P1 and delivered frozen contained in vials of $>5 \times 10^{5}$ cells $/ \mathrm{mL}$. HBEpiCs had been characterized by immunofluorescence with antibodies specific to CK18/19, and vimentin. Cells were cultured in a 1:1:1 v/v mixture of Dulbecco's modified Eagle's medium (DMEM) (Sigma-Aldrich, UK), complete mesenchymal stem cell 
medium (MSCM) (ScienCell, California, USA) and complete bronchial epithelium cell medium (BEpiCM) (ScienCell, California, USA). Cells were used in fifth passage (P5).

Human bronchial fibroblast cells (hBFs) (ScienCell, California, USA) were used for co-culture experiments. HBFs were isolated from human bronchus tissue, cryopreserved at P1 and delivered frozen in vials containing $>5 \times 10^{5}$ cells $/ \mathrm{mL}$. HBFs had been characterized by immunofluorescence with an antibody specific to fibronectin. HBFs were negative for HIV-1, HBV, HCV, mycoplasma, bacteria, yeast and fungi. Cells were cultured in minimum essential medium (MEM) (Sigma-Aldrich, UK) supplemented with $10 \%$ foetal bovine serum (FBS), 2 mM L-glutamine, $26 \mathrm{mM}$ sodium bicarbonate, $100 \mathrm{U} / \mathrm{mL}$ penicillin/streptomycin, and $1 \mathrm{mM}$ sodium pyruvate (Sigma-Aldrich, UK). This culture mixture was termed as EME. Cells were maintained at $37^{\circ} \mathrm{C}$ and $5 \% \mathrm{CO}_{2}$ in a humidified atmosphere. Cells were used in P5.

Human mesenchymal stem cells were obtained from bone-marrow (hBM-MSCs) (ScienCell, California, USA), cryopreserved at P1 and delivered frozen in vials containing $>5 \times 10^{5}$ cells $/ \mathrm{mL}$. HBM-MSCs had been characterized by immunofluorescence with antibodies specific to CD73/90/105. HBM-MSCs were negative for HIV-1, HBV, HCV, mycoplasma, bacteria, yeast and fungi. Cells were cultured in complete MSCM and maintained at $37^{\circ} \mathrm{C}$ and $5 \% \mathrm{CO}_{2}$ in a humidified atmosphere. $\mathrm{HBM}-\mathrm{MSCs}$ were also used in P5 for co-culture experiments.

\subsubsection{Seeding cells on 3D-TIPS transwell inserts}

Scaffolds (16 mm diameter, $1 \mathrm{~mm}$ thickness) were clipped to CellCrown ${ }^{\text {rm }}$ polycarbonate transwells (Scaffdex Oy; Tampere, Finland) for 24-well plates (Scheme 1B).

For monoculture conditions (Scheme 1C), $1.2 \mathrm{~mL}$ of BEpiCM medium was added into each well. 3D scaffold inserts (3D-TIPS or 3D-TIPS+Collagen) were placed into the wells and the upper chamber was filled with $500 \mu \mathrm{L}$ of Pneumacult ${ }^{\text {tm }}$-ALI medium (STEMCELL technologies; Cambridge, UK). The scaffolds were seeded at $5 \times 10^{4}$ cells $\left(2.5 \times 10^{5}\right.$ cells $\left./ \mathrm{cm}^{3}\right)$ and cultured under BEpiCM medium for 3 days to promote cells attachment. After day 3, the scaffolds were "air-lifted" by replacing $1.2 \mathrm{~mL}$ of Pneumacult ${ }^{\mathrm{T}}$-ALI medium to the basal chamber only, while the upper chamber was empty to let cells be exposed to air. Finally, medium in the lower chamber was replaced every 2 days.

For co-culture conditions (Scheme 1D), $2 \times 10^{4}$ cells $\left(9 \times 10^{4}\right.$ cells $\left./ \mathrm{cm}^{3}\right)$ of either hBFs or hBM-MSCs were first plated onto 3D-TIPS+Collagen scaffold inserts and cultured under EME or MSCM respectively for 3 days to promote cell acclimatization and attachment. Subsequently, hBEpiCs were then seeded as described above. Samples were cultured in a 1:1 mixture of medium (BEPiCM-EME or BEPiCM-MSCM). 
Polyethylene terephthalate (PET) transwell inserts were used as controls. In a 24-well plate, $500 \mu \mathrm{L}$ of BEpiCM was added to each well. A Falcon ${ }^{\bullet}$ PET filter membrane with $1.0 \mu \mathrm{m}$ pore size (Becton Dickinson Labware; Claix, France) was used as positive control and placed into the wells. The upper chamber was then filled with $200 \mu \mathrm{L}$ of BEpiCM medium. $1.8 \times 10^{4}$ cells $\left(1 \times 10^{5}\right.$ cells $\left./ \mathrm{cm}^{2}\right)$ were plated on to PET inserts to promote cell proliferation and growth until nearly confluent, according to the manufacturer's instructions. PET inserts with adhered cells were cultured under Pneumacult' ${ }^{\text {'m }}$-ALI medium conditions for 3 days to promote cell attachment. After day 3, the PET membranes were "air-lifted" by replacing 500 $\mu \mathrm{L}$ of Pneumacult ${ }^{\text {t'm}}-\mathrm{ALI}$ medium to the basal chamber only, while the upper chamber was left empty to allow the cells to be exposed to air.

\subsubsection{Cellular metabolic activity and proliferation}

Media was replaced every two days, and the metabolic activity of cells on the scaffolds ( $n=6$ per type) was monitored on days $1,3,7,10$ and 14 to determine cell viability through alamarBlue (Serotec Ltd., UK) testing. At each day point, total DNA content of the cell-laden scaffolds ( $n=6$ per type) was also quantified using a fluorescent Hoechst 33258 stain (Sigma-Aldrich, UK).

\subsubsection{Immunocytochemistry}

Immunofluorescent staining was carried out to detect the presence of various markers of epithelial differentiation and functionality: MUC5AC, ZO-1, FOXJ1, Ki67, p63, vimentin, keratin 5, keratin 14, keratin 18 , acetylated $\alpha$-tubulin, e-cadherin and f-actin. Cell-laden scaffolds and cell inserts were washed in Dulbecco's Phosphate Buffered Saline (DPBS; ThemoFisher, UK) and fixed in cold 4\% PFA in PBS up to $18 \mathrm{~h}$ overnight. Samples were permeabilized with $0.1 \%$ Triton-X 100 (Sigma-Aldrich, UK) for $15 \mathrm{~min}$, rinsed with DPBS and blocked with $1 \%$ bovine serum albumin (BSA) (Sigma-Aldrich, UK) in PBS solution for $30 \mathrm{~min}$. Following further rinsing, they were then incubated with various fluorescent dyes and conjugated antibodies. A complete list is shown in Table S1. Finally, samples were mounted in Fluoroshield ${ }^{\circ}$ with DAPI (Sigma-Aldrich, UK). Images were taken and analyzed using a confocal microscope (Leica Spvi8, Germany). The total counts per field of view $(560 \mu \mathrm{m} \times 560 \mu \mathrm{m})$ was not less than 10 frames per scaffold ( $\mathrm{n}=3$ per type), and 500 cells were recorded and analyzed using ImageJ (version 1.47; NHI, USA).

\subsubsection{Cell morphology by scanning electron microscope (SEM) and focused He-ion based microscope (He-ion Fib) \\ Cell-laden scaffolds were imaged using SEM/Fib to assess epithelial cell morphology and monolayer} formation on the surface of the scaffolds ( $n=3$ per type). Samples were fixed in $3 \%$ glutaraldehyde (Sigma-Aldrich, UK) for $1 \mathrm{~h}$ at room temperature, dehydrated in a series of ethanol solutions and dried using supercritical carbon dioxide in a critical point dryer. Dry constructs were sputter-coated with gold 
(10 nm thickness) and imaged using Zeiss XB1540 Fib/SEM and Zeiss Orion NanoFab. Low magnification images were captured using XB1540 Fib/SEM at $1 \mathrm{kV}$ and working distance of $3.5 \mathrm{~mm}$, while high magnification images were taken using Orion NanoFab He-ion FIB at $25 \mathrm{kV}$ and working distance of about $7 \mathrm{~mm}$.

\subsubsection{Quantitative reverse-transcriptase polymerase chain reaction (qPCR)}

Relative gene expression of epithelial cells seeded on the scaffolds was quantified using qPCR. Briefly, total RNA was collected and extracted from samples ( $n=5$ per scaffold type) in triplicate using Qiagen's RNeasy mini kit (Qiagen; Valencia, CA) according to the manufacturer's instructions on weeks 1, 2 and 3. Samples were treated with DNase to remove genomic contaminants and RNA was then reversetranscribed to cDNA using an iSCRIPT cDNA synthesis kit (BioRad, USA). The relative expression of genes was then determined via real-time PCR (BioRad, USA). Markers of epithelial primer assays (Qiagen, Valencia, CA) used are listed in Table S2, where glyceraldehyde-3-phosphate dehydrogenase (GAPDH) served as an endogenous control "housekeeping gene" for all samples.

\subsubsection{ELISA analysis}

Enzyme-linked immunosorbent assay (ELISA) was used to detect presence of MU5AC, FOXJ1, ZO-1 and keratin 18 in scaffolds ( $n=4$ per type) within their cell culture medium at weeks 1, 2 and 3 . ELISA kits used were MUC5AC (Abbexa, UK), TJP1 (ZO-1) (antibodies-online, UK), FOXJ1 (antibodies-online, UK), and keratin 18 (antibodies-online, UK). Optical density was determined using a microplate reader (Anthos 2020; Biochrome Ltd, UK).

\subsubsection{Immunohistochemistry}

Cell-laden scaffolds ( $n=2$ per type) were fixed in $4 \%$ paraformaldehyde (PFA) (Sigma-Aldrich, UK) in phosphate buffered saline (PBS), embedded in paraffin wax and cut into $4 \mu \mathrm{m}$ thick sections using a Leica RM2235 microtome (Leica Microsystem Ltd.; Milton Keynes, UK). Hematoxylin and eosin (H\&E) staining was performed to examine gross cell location and morphology, and Alcian Blue-periodic acid Schiff (AB-PAS) staining to identify cells with mucosal substances and observe cell distribution and migration into the scaffolds. Images were taken using a digital slide scanner (Leica SCN400F, Germany) at $\times 40$ magnification.

\subsubsection{Transepithelial electrical resistance (TEER) measurements}

The integrity of the epithelial barrier formed by hBEpiCM cells cultured on the scaffolds was quantified by the measurement of TEER in monoculture and co-culture conditions. Prior to measurement of TEER using an EVOM voltohmmeter (World Precision Instruments; Stevenage, UK), cell culture medium was initially added to the apical compartment of the ALI cultures and samples ( $n=6$ per scaffold type) were 
incubated for $1 \mathrm{~h}$. Electrical resistance was measured using STX-2 chopstick electrodes (World Precision Instruments; Stevenage, UK) immediately upon removal of cells from the incubator. TEER ( $n=6$ measurements per sample) was calculated by subtracting the resistance of a cell-free scaffold or insert and correcting for the surface area available for epithelial cell growth $\left(0.39 \mathrm{~cm}^{2}\right)$. To compare TEER values between groups following a plateau of the measurements [29], the average TEER values from day 14 were taken for each group and compared.

\subsubsection{Fluorescein isothiocyanate (FITC)-labeled dextran 70 (FD70) permeability assay} The integrity of the epithelial barrier formed by hBEpiCs on the scaffolds ( $n=6$ per type) was further assessed by analysis of FD70 paracellular transport through the cell layer [30]. Samples were initially washed and incubated with Hank's buffered salt solution (HBSS) (Sigma-Aldrich, UK) in both the apical and basolateral compartments for $0.5 \mathrm{~h}$. Subsequently, the buffer in the apical compartment was replaced with a $500 \mathrm{mg} / \mathrm{mL}$ solution of FITC-labelled dextran of an average molecular weight of $70 \mathrm{kDa}$ (FD70), and the sampling from the basolateral compartment was performed every $20 \mathrm{~min}$ for $1 \mathrm{~h}$ to quantify transported drug ( $n=6$ measurements per sample). An equal volume of buffer was used to replace the removed volume of basolateral solution at each time point. Additionally, a sample of the initial apical FD70 content was taken for analysis and TEER measurements were performed before and after the experiment to validate that the barrier integrity was unaltered during the transport assay. The fluorescence of sampled time points was quantified by measuring excitation at $485 \mathrm{~nm}$ and emission at $535 \mathrm{~nm}$. Fluorescence values were converted to concentration of FD70 using a standard curve and the apparent permeability coefficient (Papp) of FD70 was calculated (Eq. 1):

$$
\operatorname{Papp}\left(\mathrm{cm} \cdot \mathrm{s}^{-1}\right)=F /\left(A^{*} C_{0}\right)
$$

where $F$ is flux (i.e. rate of change in cumulative mass transported), $A$ is surface area of the cell culture support, and $\mathrm{C}_{0}$ is the initial concentration in the donor chamber.

The theoretical pore radii of the cell layers was estimated by calculating the Renkin function [31] (Eq. 2):

$$
r_{i} / r_{p}=\left[1-\left(r_{i} / r_{p}\right)\right]^{2} *\left[1-2.109\left(r_{i} / r_{p}\right)+2.09\left(r_{i} / r_{p}\right)^{3}-0.95\left(r_{i} / r_{p}\right)^{5}\right]
$$

where $r_{i}$ is the molecular radius of the solute $(n m)$ and $r_{p}$ is the theoretical pore radius $(n m)$.

\subsection{Data analysis}

Results were presented as standard deviation (SD, error bars) of the mean values. Statistical analysis was performed using Graph-Pad Prism 6 (GraphPad, USA), by one-way (one independent variable) or two- 
way (more than two independent variables) analysis of variance (ANOVA) using Tukey's post hoc test for multiple comparisons. A value of $p<0.05$ was considered statistically significant.

\section{Results}

\subsection{Morphology and stiffness softening of 3D-TIPS hybrid scaffolds} SEM morphology of the surface of the 3D-TIPS scaffold (Fig. 1A1a) shows a digitally defined interconnected macro-porous structure using the 3D-TIPS process, a combination technique of inverse 3D printing and phase separation suitable for manufacturing unprintable solution based polymers or nanocomposites [24]. Different from direct printing, the porous structures consisting of micro- to nanopores, as well as a highly ordered nano-phase structure of PUU-POSS (Fig. 1A1b), were generated during simultaneous coagulation and nano-phase separation of the soft and hard segments of block copolymer PUU with POSS terminals within the micro-channels of the directly 3D printed PVA preform in water. Such a highly interconnected porous structure provides a large surface area to adsorb and immobilize the collagen solution. As a result, coating of collagen hydrogel (Fig. 1A2a-A2b) provided an additional nanofibrous structure, interpenetrating throughout the interconnected printed network, mimicking more closely the characteristic structure and function of the extracellular matrix of trachea $[32,33]$.

The mechanical properties and viscoelasticity of the collagen coated and uncoated 3D-TIPS scaffolds were characterized and summarized in Fig. 1B-D. Obviously, the addition of the collagen hydrogel network within the 3D-TIPS scaffold did not result in any significant changes in terms of the strength and modulus through static tensile and compression tests, owning to its weaker mechanical properties than the PUU-POSS scaffold alone (Fig. 1B1). The stiffness softening properties at body temperature previously reported [24] were observed again in both static (Fig. 1B2) and dynamic testing modes (Fig. 1C1-D2). After a 28-day period of isothermal relaxation at $37^{\circ} \mathrm{C}$, a decrease in both tensile and compression modulus was exhibited (Fig. 1B2). Stiffness softening was manifested under cyclic tensile and compression loadings over 200,200 cycles at a fixed 25\% strain (Fig. 1C1-D2), with evidence of a pronounced reduction of both the load amplitude and the hysteresis loop area, indicating that the scaffold became softer with increasing reversible compliance during the stiffness damping effect. The initial high stiffness of the scaffold was contributed by the formation of localized crystalline structure of the soft segments of PUU within its nanophase structure (Fig. 1A2b) during the freezing process of the polymer solution in the 3D printed PVA mold, which was also observed in our previous work [24]. Stiffness softening is contributed by the relaxation of 3D-ordered crystalline structure of the soft segments at body temperature. 


\subsection{Epithelial cell growth on 3D-TIPS scaffolds}

Cell seeding density onto a new scaffold is critical for initial cell attachment and migration. For this reason, the optimization of the cell seeding process was initially performed using hBEpiCs at three different cell seeding densities $\left(0.8 \times 10^{4}, 3 \times 10^{4}\right.$ and $5 \times 10^{4}$ cells/scaffold $)$ under monoculture conditions. Fig. 2A shows the cell seeding efficiency of hBEpiCs onto 3D-TIPS scaffolds over a 14-day period. Reduced metabolic activity was seen at $0.8 \times 10^{4}$ and $3 \times 10^{4}$ cells/scaffold during cell culture. Despite a slight reduction on day 10 , the optimal cellular viability at $5 \times 10^{4}$ cells/scaffold remained increasing over time, which was chosen as the cell seeding parameter for further study. HBEpiCs cultured on collagen hydrogel functionalized composite scaffolds (3D-TIPS+Collagen) exhibited significantly greater metabolic activity (Fig. 2B) and proliferation (Fig. 2C) over a 10-day period, compared to the untreated 3D-TIPS scaffolds $(p<0.001)$.

Immunofluorescence micrographs (Fig. 2D-H) show that a majority of hBEpiCs retained undifferentiated basal cells (keratin 5 [34]), meanwhile expressed markers for supporting epithelial differentiation (vimentin) and stratified epithelium (p63 as transcriptor [35]), and intercellular junctions (f-actin bundles) were observed on the periphery of hBEpiCs on all scaffolds at day 7, with p-value nonsignificant. In contrast, hBEpiCs on 3D-TIPS+Collagen scaffolds co-cultured with hBM-MSCs exhibited less percentage of stained mitotic cells (Ki-67 marker, Fig. 2D), indicating less DNA synthesis, compared to their co-culture with hBFs ( $p$-value non-significant) and the untreated 3D-TIPS scaffold $(p<0.01)$. More actin filaments protruded to the edge of the cell membrane to propel cell attachment, movement and immigration by extending their lamellipodia around the edges of the cells to adhere to each other and to those functionalized scaffolds. Better formation of lamellipodia (highlighted by white circles, Fig. 2E5H5) were observed in close-up confocal microscopic images of basal cell marker (keratin 5) on the scaffolds with collagen hydrogel and co-cultured with hBFs and hBM-MSCs, compared to the untreated 3D-TIPS scaffolds. HBEpiCs also expressed more vimentin (Fig. 2E6-H6) on those scaffolds. SEM images in Fig. 3A1-D1 provide more information about cell morphology and the interface between the cells themselves and the scaffolds. HBEpiCs on the collagen functionalized and co-cultured scaffolds look flatted and connected to each other, even merged with blurred boundary on the hBM-MSC co-culture, as opposed to the round shape with less actin filaments protruding and connections between the cells on the untreated 3D-TIPS scaffolds.

\subsection{Epithelial ciliation in scaffold culture}

3D-TIPS and 3D-TIPS+Collagen scaffolds were assessed for their ability to support hBEpiCs growth and differentiation, either as monoculture or in co-culture with hBFs or hBM-MSCs under air-liquid interface 
(ALI) conditions (Scheme 1). The formation of motile cilia in the various scaffold cultures was assessed through FOXJ1 (marker of epithelial cell ciliation) analysis of gene expression (Fig. 4A1) and ELISA analysis of FOXJ1 over a 3-week period (Fig. 4A2). HBEpiCs mono- and co-cultured on the 3DTIPS+Collagen scaffolds showed significant upregulation of FOXJ1 gene expression compared to the untreated 3D-TIPS scaffold and PET control for two weeks, indicating that 3D-TIPS+Collagen scaffolds promote more sustained increase in gene expression. More cilia were highlighted by acetylated alpha tubulin staining both in-plane and cross-section of 3D-TIPS+Collagen scaffolds, compared to the untreated 3D-TIPS or PET, and even much more extent in co-culture conditions at day 21 (Fig. 4B1-E1). A similar trend of formation of pseudostratified columnar epithelial cell arrays was also observed. FIB images (Fig. 5A-E) further revealed a dense and long pseudostratified columnar morphology on the collagen functionalized and co-cultured scaffolds (Fig. 5C1-E4), in contrast to the sparse dot-like morphology in cultures with PET cell inserts (Fig. 5A1-A4). Cross-sectional images by He-ion beam FIB (Fig. 5F) confirmed that an epithelial layer had covered the scaffold with more prominently longer and thicker ciliary structures formed on 3D-TIPS+Collagen scaffolds, especially when co-cultured with hBMMSCs (Fig. 5G-H, Table S3).

\subsection{Epithelial barrier formation and mucin expression on 3D-TIPS scaffold.}

The ability of the scaffolds to support hBEpiC cell differentiation was also assessed by analysing the expression of the tight junction protein ZO-1 (also known as occludin-1) and adherens junction of associated protein, e-cadherin, as markers of bronchial epithelial barrier formation. HBEpiCs mono- and co-cultured on 3D-TIPS+Collagen scaffolds exhibited an upregulation of ZO-1 gene expression $(p<0.0001)$

(Fig. 4A3) and ELISA analysis (Fig. 4A4) ( $p<0.05)$ significantly higher in co-culture with hBM-MSCs, compared to the untreated 3D-TIPS or PET control. Formation of both tight junctions (ZO-1 in red, Fig. 4B2-F2) and adherens junctions (e-cadherin in green, Fig. 6B1-F1) were visualized at day 21 through immunofluorescent images. Well-stratified tight junction columnar (ZO-1 in red, Fig. 4B1-1 to F2-1), and adherens columnar junctions (e-cadherin in green, Fig. 6B1-1 to F1-1) were vertically aligned under the epithelium for both co-culture conditions, compared to the monocultures. In particular, adherens junctions (Fig. 6E1-1 and F1-1) were well structured and connected in co-culture condition, and even extended into the supporting layer of hBM-MSCs, opposed to the disoriented and disconnected ones in monoculture conditions.

Mucin 5A (MUC5AC) is the gene associated with goblet cells that secrete mucin in the respiratory epithelium. Analyses performed by qPCR and ELISA (Fig. 6A1-A2) showed that MUC5AC expression is upregulated on 3D-TIPS+Collagen scaffolds in both mono- and co-cultured conditions for three weeks 
$(p<0.0001)$, compared to the untreated 3D-TIPS and PET control. The gene expression also significantly differentiated between co-cultures with hBFs or hBM-MSCs compared to monocultures $(p<0.001)$. Immunofluroescent staining of expressed MUC5AC glycoprotein at day 21 (red, Fig. 6B1-F1-1) corroborated the above quantitative analyses, showing more sustained mucus-secreting epithelial phenotype on the co-cultured samples, compared to the monocultured scaffolds, with significantly more on hBM-MSCs co-culture (Fig. 6F1-1). Overall, these results provide more clear evidence that hBEpiCs formed a functional epithelial barrier on the 3D-TIPS+Collagen scaffolds cultured at an air-liquid interface. Co-culture with hBM-MSCs significantly enhanced the stratification of epithelium by the formation of more in-depth well connected adherens junctions and more goblet cells.

\subsection{Analysis of basal cell and differentiated cells}

The expression of intracellular keratin 14 and intracellular keratin 18 indicated the organization of differentiated mitotically active basal epithelial cells and differentiated cells in the epithelium on the various scaffolds. QPCR and ELISA analysis showed that keratin 18 expression is upregulated on 3DTIPS+Collagen scaffolds in both mono- and co-culture, compared to untreated 3D-TIPS (Fig. 6A3-A4), and reached the highest on week 2 when co-cultured with hBM-MSCs ( $p<0.001$, Fig. 6A4). Undifferentiated hBEpiCs at day 7 were stained with keratin 5 (Fig. 2-3), and differentiated hBEpiCs at day 21 (Fig. 6B2-F2) were stained with keratin 14 as marker of basal cells, and keratin 18 as a marker of differentiated cells for distinguishing the basal and differentiated layers within the epithelium. As expected, at day 21 keratin 14 was mostly visible on the basal layer at the cell-scaffold interface (Fig. 6B2-1 to F2-1), while keratin 18 was present on the upper layer of the epithelium for all groups (Fig. 6B2 to F2 and B2-2 to F2-2). More staining for both keratin markers were seen on 3D-TIPS+Collagen scaffolds compared to the control or untreated 3D-TIPS. Cross-sectional images (Fig. 6B2-2 to F2-2) showed a thicker epithelium for co-culture conditions with hBM-MSCs where more keratin 18 appeared on the apical layer, indicating that more basal cells differentiated into ciliated and secretory cells. Histological examination of 21-day cultured scaffolds (Fig. 7A-E) shows well-developed ciliated cells and mucosubstance by Alcian blue-Periodic acid-Schiff (AB-PAS) (magenta at arrow) on the scaffolds. An upper ciliated monolayer appeared more prominent on 3D-TIPS+Collagen scaffolds compared to the untreated 3D-TIPS and PET control, and the highest number of mucus-secreting cells were observed on co-culture scaffolds with hBM-MSCs, resembling that of native bronchial epithelium. Further quantification of percentage of cell counts (Fig. 7F) stained by F-actin and AB-PAS also agreed with those by the GPCR and ELISA analyses above (Fig. 4 and Fig. 6). It is of note that the ciliated layer was well integrated with porous structure of the scaffolds showing more cells migration and ingrowth across 
most of pores in both co-culture conditions (Fig. 7D1 and 7E1). In contrast, the delicate cilated layer was valuable and easily delaminated from PET substrate fixed in transwell insert (Fig. 7A1).

\subsection{Epithelial barrier integrity in scaffold co-culture}

Transepithelial electrical resistance (TEER) measurements (Fig. 8A) were used to confirm an effective barrier function of the differentiated epithelial layer on all the scaffolds with mean TEER values of 496 ( \pm 53$), 972( \pm 211), 1110( \pm 320)$ and $1187( \pm 230) \Omega \mathrm{cm}^{2}$ on day 14 for monoculture on 3D-TIPS, monoculture on 3D-TIPS+Collagen and co-cultures on 3D-TIPS+Collagen scaffolds with hBFs or hBMMSCs respectively (Table S4). TEER values increased dramatically in co-culture with hBFs or hBM-MSCs, despite being non-significant between them. The ability of the epithelial barrier to impede paracellular transport of FD70 was evaluated by Papp values in all scaffolds (Fig. 8B, Table S5), showing that cells cultured on 3D-TIPS+Collagen were less permeable to solute flux than cells cultured on untreated 3DTIPS. The Papp values and average molecular weight of the compound can be employed to determine the theoretical equivalent pore radii of the cell layer. The epithelial layer in co-culture with hBFs or hBMMSCs exhibited smaller pore radii of the cell layers ( $5.2 \mathrm{~nm}$ and $4.1 \mathrm{~nm}$ respectively) compared to monoculture conditions on PET, 3D-TIPS or 3D-TIPS+Collagen ( $65 \mathrm{~nm}, 62 \mathrm{~nm}$ and $13 \mathrm{~nm}$ respectively). Diffusion transport of FITC-dextran showed a dramatic reduction from the basolateral to apical on 3DTIPS+Collagen with monoculture and co-culture conditions, in contrast to the diffusion from the apical to basal which remained fairly constant in Fig. 8C-D. Therefore, the decrease of the permeability for 3DTIPS+Collagen scaffolds was mainly determined by the diffusion from the basolateral to apical, and the asymmetric diffusion through the cell layers for the two opposite diffusion directions was minimized through the better growth and maturation of the epithelium layer.

\section{Discussion}

The dynamic thermoresponsive stiffness softening behaviour of the scaffolds were reproducibly demonstrated. The unique stiffness softening of the soft elastomer nanohybrid scaffolds at human body temperature induced by 3D-TIPS process is summarized in Fig. 1. A more systematical mechanical property study of the 3D-TIPS scaffolds and as 2D-TIPS foam structures had been reported previously [24-27]. Such thermoresponsive property was driven by the phase transition from the semicrystalline phase (Fig. 1A1-b) to rubber phase and reverse self-assembling of the quasi-random nanophase structure when subjected at body temperature near the melting point of the crystalline domains of the soft segments of PUU [24-27]. PUU-POSS is chemically stable and non-degradable, similar to other polyurethane elastomers. The gradual reduction of the stiffness measured at culture time was proven to be attributed to the physical relaxation of ordered crystalline polymer chains $[24,26]$. The stiffness 
softening of these scaffolds, coupled with their interconnected micro/nano- porous structure, facilitated cellular growth of hDFs [24] and modulated the differentiation of hBM-MSCs towards chondrogenesis and osteogenesis when cultured in differentiation media [25]. Our recent in vivo study further showed that the stiffness relaxation mechanism exhibited by these 3D-TIPS 3D-printed scaffolds could modulate tissue ingrowth and promote good vascularization guided throughout the digitally defined network in rat models for up to 3 months with a reduced inflammatory response [27]. These recent achievements pave the way here for the further development of more complex multi-layered tracheobronchial epithelium-like tissues in vitro based on these thermoresponsive soft scaffolds.

In particular, tracheal wall tissue consists of a distinct layered composite structure: ciliated columnar epithelium luminal surface, C-shape cartilage rings joint with soft connective tissue and with smooth muscle fibres. The cartilage rings predominately contribute the structure stiffness of the tracheal tube. In humans, the mechanical properties of the tracheal cartilage are gender and age dependent, with the tensile Young's modulus reported in the range of 1-25 MPa [36-38]. Taking into consideration that the porcine trachea model was generally believed to resemble more closely that of human, the compression modulus of the porcine's trachea has been reported in the range of 1-5 MPa $[39,40]$. It is of note that the tensile modulus of the porous 3D-TIPS scaffolds with $50 \%$ infill density $(\sim 1 \mathrm{MPa})$ is close to the low end of these tissue values and the decellularized rat trachea scaffolds ( $\sim 939 \mathrm{kPa})$, but much higher than collagen hydrogel (1-10 kPa) [41-43]. The tensile modulus of the 3D-TIPS scaffolds can be obtained in the range of 0.7 to $4.4 \mathrm{MPa}$ by varying the infill density and processing conditions [24]. However, it is of note that, in the case of the $50 \%$ infill density with the desired porosity in this study, the overall structure stiffness of a tubular construct made from highly porous 3D-TIPS alone may not be stiff enough to meet the requirements for human airway reconstruction.

Nevertheless, the outstanding compliance and robustness of these thermoresponsive 3D-TIPS scaffolds in both static and dynamic condition at human body temperature provide a promising matrix for soft tissue engineering. The initial higher stiffness of the scaffold will be another bonus to provide mechanical support in the early stages of cell growth and post-surgical healing. The scaffold becomes even softer after stiffness relaxation while retaining outstanding hyperelasticity. Therefore, the stiffness softening will promote enhanced integration with the surrounding tissues, with better match to the dynamic remodelling of stiffness of the connective tissue and muscle, as well as potential vascularization in vivo [27]. The resulting tensile modules of the explant show a significant increase up to $6.97 \mathrm{MPa}$ after subcutaneous implantation for 12 weeks, which is in the range of human the tracheal cartilage with the tensile Young's modulus (1-25 MPa) aforementioned [36-38]. This is also supported by the histological structure of strong interface between epithelial tissue layer and the scaffold, in contrast to the 
delaminated delicate epithelial layer on rigid PET substrate in transwell insert (Fig. 7). Moreover, the extended chondrogenic differentiation period ( 35 days in vitro) after stiffness softening confers the scaffolds cartilage-like compression mechanical properties worthy of being considered for tracheal tissue engineering [25], still lower than the compression modulus of the porcine's trachea in the range of 1-5 MPa, but close to femoral non-mineralized and mineralized fibrocartilage exhibiting compressive Young's moduli in the range $0.55-0.80 \mathrm{MPa}$ and $0.20-0.60 \mathrm{MPa}$ respectively.

To improve hBEpiCs viability, these scaffolds that served as a primary substrate were further functionalized by impregnating a collagen hydrogel within the interconnected porous network of the synthetic PUU-POSS elastomer, with proven benefit of collagen in respiratory epithelial culture $[11,21,44,45]$. The coating of collagen hydrogel does not affect the static hyperelastic mechanical properties of the 3D-TIPS scaffolds (Fig. 1B1). The systematic study on hBEpiCs cell growth and differentiation on the functionalized hybrid scaffolds in both monoculture and co-culture conditions have elucidated the capacity of such scaffolds to support an epithelium bioprocess in vitro with formation of a tissue-like architecture, physiologically relevant to 3D human tracheobronchial biofunctions. The results provide coherent evidence of hBEpiCs' growth, expression of mucin and cilia and formation of an epithelial barrier with cell retention at the air-liquid interface supported by the scaffolds.

Cilia are an important feature of a fully-functional tracheobronchial epithelium since they are an integral component of the respiratory tract that assist with removal of particulates and debris from the airway [46]. 3D-TIPS+Collagen scaffolds were demonstrated to support the expression of cilia in hBEpiCs, in evidence of significant upregulation of FOXJ1 (a master regulator of motile cilogenesis [46]) at several orders of magnitude higher than that on PET control ( $p<0.0001$, Fig. 4A1) in a short period of time (14 days). This suggests an earlier promotion of cilogenesis in hBEpiCs cultured on 3D-TIPS+Collagen scaffolds since cilogenesis typically takes around 21 to 28 days to develop [47]. Microvilli (stained by acetylated alpha tubulin, Fig. 4B1-F1) formed on 3D-TIPS+Collagen scaffold monoculture were longer ( $\sim \mu \mathrm{m}$ in length) and thicker in shape compared to the untreated 3D-TIPS ( $\sim \mu \mathrm{m})$ and PET control ( 2 $\mu \mathrm{m})$ (Fig. 5G-H, Table S3). The average cilia length in a human airway (healthy non-smoker individual) has been reported to be $\sim 5 \mu \mathrm{m}$ [48], but only $\sim 0.5 \mu \mathrm{m}$ on PET control and $\sim 1 \mu \mathrm{m}$ for human bronchial epithelial Calu-3 cell monocultures on bi-layered collagen-hyaluronate scaffolds [21]. In comparison with ciliated Calu-3 cells used in collagen-hyaluronate scaffolds with/without retinoic acid drug [21,49,50], the cilia grew significantly longer on only collage coated 3D-TIPS scaffold in both mono- and co-culture conditions, indicating higher potential for ciliation of human bronchial epithelial cells on the top of such 3D printed porous network. 
MUC5AC glycoprotein is a substantial component of the respiratory mucus coating and an indicator of mucociliary epithelial cell differentiation [51,52]. The significant increase of the expression of MUC5AC gene (Fig. 6A1) on 3D-TIPS+Collagen scaffolds manifests more goblet cells differentiation, which may be contributed by the presence of collagen network within the scaffolds [53]. The mucus secreted by goblet cells is an important biofunction of the pulmonary tract with a prominent role as defense barrier. However, it can hinder delivery of aerosolized therapeutics to the epithelium or down the airway to the alveolar region [54]. A previous study had reported that sufficiently small enough particles $<500 \mathrm{~nm}$ could rapidly penetrate the respiratory mucus as opposed to those of larger size [54]. Therefore, the ability of the 3D-printed hybrid scaffolds to induce greater mucus secretion is of great value for disease modeling to help design and test upcoming therapeutic mucus-penetrating nanoparticles for airway delivery. 3D-TIPS+Collagen scaffolds also enhanced the expression of tight junction associated cytoplasmic scaffolding protein ZO-1 (Fig. 4A4) and adherens junction associated transmembrane protein e-cadherin (Fig. 6B1-F1), indicating the formation of an epithelial barrier layer. The visualized intercellular mesh-like network of tight junctions and adherens junctions are characteristic of cell-cell adhesion involved in reorganization of the actin cytoskeleton in epithelial monolayers, similar to Calu-3 cells [30]. The two junctions provide different functions, with adherens junctions initiating cell-cell contacts and mediating the maturation and maintenance of the contact, and tight junctions regulating the paracellular pathway for the movement of ions and solutes in between cells [55]. Their localization in the cell periphery and their affiliation with ZO-1 protein and e-cadherin are recognized as a core component of the barrier integrity [56]. In addition, detection of f-actin on the hBEpiCs circumference reinforced the hypothesis of the presence of an epithelial barrier (Fig. 2E2-H2).

The capacity of the 3D-TIPS hybrid scaffolds was substantially boosted by an epithelial fibroblast-like coculture model: hBEpiCs co-cultured with either hBFs or hBM-MSCs outperformed the monoculture model. It is of note that the digitally printed porous architecture of the 3D-TIPS scaffolds has been demonstrated to promote cell migration and proliferation with their own deposited ECM components $[24,25]$, as well as to guide the growth of vascular capillary network in vivo [27]. This is further testified that the thin epithelial layer was closely integrated with co-cultured cells growing within interconnected pores of the scaffolds. Gene expression of the markers of mucus secretion (MUCA5AC), barrier formation (ZO-1) and ciliation (FOXJ1) in hBEpiCs and related mRNA translated proteins were all accelerated in the co-cultures, reaching the highest with hBM-MSCs. More goblet cells (marked by MUCA5C) and fully elongated cilia (marked by acetylated alpha tubulin, Fig. 4B1-F1) were observed in both co-culture scaffolds, but more prominence, in terms of cilia length and diameter, was observed by FIB in co-cultures with hBM-MSCs (Fig. 5G-H). The differentiated epithelial layer co-cultured with hBM- 
MSCs consisted of $\sim 36 \%$ goblet cells, $\sim 55 \%$ ciliated cells and $\sim 9 \%$ non-ciliated cells, a step closer to the human airway epithelium of a healthy individual, reported as $\sim 20 \%$ goblet cells, $\sim 60 \%$ ciliated cells and $\sim 20 \%$ basal cells $[57,58]$ (Fig. 7F). Both tight junctions and adherens junctions were well-constructed to hold stratified epithelium, in particular, adherens junctions grew deeper, integrating tightly with the hBM-MSC supporting layer. Overall, these results demonstrated that co-culture with either hBFs or hBM-MSCs on 3D-TIPS+Collagen scaffolds have promoted more initiation and stabilization of cell adhesion, regulation of the actin cytoskeleton, intracellular signaling and transcriptional regulation during epithelium formation. Such enhancements could be postulated that a culmination of signaling events regulated by both the scaffold and cellular factors, especially from hBM-MSCs, could substantially increase cell-cell contact and adhesion, and accelerate the formation, maintenance and function of the epithelium barrier.

TEER results provide a measure of the integrity and permeability of the epithelial layer. TEER values from ex vivo human lung tissue have not been reported so far, but it has been shown to exhibit a range of 300 to $650 \Omega \mathrm{cm}^{2}$ on rabbit tissue and human primary epithelial cell cultures [59]. Higher TEER values (around $900 \Omega \mathrm{cm}^{2}$ ) had been previously reported on bi-layered collagen-hyaluronate scaffolds using Calu-3 epithelial cells in co-culture with Wi38 fibroblasts [21]. Much higher TEER values were reported here for the 3D-printed hybrid scaffolds in both mono- and co-cultured conditions (up to $1372 \Omega \mathrm{cm}^{2}$ in the case of hBEpiCs/hBM-MSCs between day 7 \&10), similarly to those on day 14 reported on bilayered collagen-hyaluronate scaffolds when loaded with retinoic acid drug using Calu-3 epithelial cells in co-culture with Wi38 fibroblasts [50]. The increase of TEER values and decrease of paracellular permeability $\left(\mathrm{P}_{\text {app }}\right)$ on the scaffolds from monoculture to co-culture conditions corroborated the development of tight junctions and adherens junctions within the epithelial layer [60] and the predicted smaller pore radii of the cell layer $[30,61]$. It is of note that the asymmetry in the diffusion was drastically diminished in the co-culture with hBM-MSCs, which may be correlated to a more uniform and aligned stratified epithelium layer tightly integrated with hBM-MSCs underneath via deeper adherens junctions. Therefore, all the results indicated that the epithelial barrier was profoundly robust in the samples with hBEpiCs/hBM-MSCs in co-culture.

More tissue engineered approaches for developing tracheobronchial epithelium have been reported recently. For instance, a 3D-printed scaffold based on silk fibroin and hydroxypropyl methyl cellulose thixotropic hydrogel [62] was developed and assessed with a human bronchial epithelial cell line. In a different study, a composite of chitosan, gelatin and poly(lactide) in a bi-layered non-woven scaffold was produced by electrospinning and its efficacy evaluated with primary airway epithelial cells [63]. 
However, it is not straightforward to compare the results reported here with these engineered tissues which were not yet fully developed with limited characterizations and analyses.

Overall, a physiologically relevant in vitro tracheobronchial epithelial model has been developed on the interpenetrated hybrid network of the 3D-TIPS+Collagen scaffolds. This study has proven the epithelial functionality in vitro of disc-shaped 3D printed scaffolds with coated collagen hydrogel in both monoculture and co-culture conditions in well plates, which is imperative for the next step of the design and manufacturing of patient's specific tracheal scaffolds. It is of note that, as shown by comparing the case of 3D-TIPS and 3D-TIPS+Collagen in monoculture conditions, epithelialization on the 3D-TIPS without the collagen component (to provide integrin-mediated cues to the epithelial cells in conjunction with embedded submucosal cells) is far less successful if compared with 3D-TIPS+Collagen. Therefore, the interpenetrated network in combination of thermoresponsive hyperelastic 3D-TIPS scaffolds with collagen hydrogel holds more promise to provide a strong, tough and biofunctional hybrid for producing tubular constructs with tunable stiffness through the structure design and 3D-TIPS manufacturing process.

The translation of these 3D-TIPS scaffolds into actual 3D tubular constructs with the same dimensions and stiffness as the patient's airway track is yet to be developed in a future study, which is another critical criterion for tracheal tissue engineering $[13,64]$. Two possible approaches to improve the overall structural stiffness of tracheal scaffolds made from 3D-TIPS scaffolds are on-going. One is to develop a composite scaffold with reinforcement of a stiffer stent acting as cartilage rings in native trachea. Another more regenerative approach is to introduce synthetic cartilage within 3D-TIPS scaffold through a co-culture of chondrogenic differentiation of hBM-MSC and epithelization of hBEpiCs. Both studies will be featured in future publications. Other than for surgical reconstruction applications, future studies will also explore these tracheobronchial epithelial models and related airway disease model for drug screening and discovery.

\section{Conclusion}

This study has developed biomimetic hybrid composite scaffolds as a 3D in vitro airway model by indirect 3D-TIPS printing. The hierarchically interconnected porous structure of 3D-TIPS elastomeric scaffolds allows collagen hydrogel to impregnate into an interpenetrated hybrid network. The epithelium presented on the scaffolds is a synergetic bioengineering combination of collagen hydrogel, a 
thermoresponsive elastomeric nanohybrid network, epithelial cell culture and bi-layered co-culture with either hBFs or hBM-MSCs underneath. The 3D hybrid scaffolds demonstrated their capacity to support the growth and differentiation of human bronchial epithelium, in addition to epithelial fibroblast-like cocultures, generating biological structures and functions close to the human respiratory tract tissue. A coculture with hBM-MSCs accelerated the maturation of the epithelium through promotion of more cellcell interaction and regulation of the actin cytoskeleton, intracellular signaling and transcriptional regulation. The 3D-TIPS printing approach offers a customizable reproducible technology to generate a physiologically relevant 3D biomimetic system to advance our understanding of airway disease and subsequent drug discovery. The thermal-mechanically and biologically responsive scaffold will serve as a future platform for personalized surgical reconstruction and regeneration, and more studies will be featured in future papers.

\section{Acknowledgements}

The authors acknowledge financial support by the Engineering and Physical Sciences Research Council (EPSRC) in the United Kingdom (grant no. EP/L020904/1, EP/M026884/1 and EP/R02961X/1).

\section{Declaration of competing interests}

The authors declare that they have no known competing financial interests or personal relationships that could have appeared to influence the work reported in this paper.

The authors declare no conflict of interests.

\section{References}

[1] S.M. May, J.T.C. Li, Burden of chronic obstructive pulmonary disease: Healthcare costs and beyond, Allergy Asthma Proc. 36 (2015) 4-10. https://doi.org/10.2500/aap.2015.36.3812.

[2] C.D. Mathers, D. Loncar, Projections of global mortality and burden of disease from 2002 to 2030, PLOS Medicine. 3 (2006) e442. https://doi.org/10.1371/journal.pmed.0030442.

[3] K. BéruBé, Z. Prytherch, C. Job, T. Hughes, Human primary bronchial lung cell constructs: The new respiratory models, Toxicology. 278 (2010) 311-318. https://doi.org/10.1016/j.tox.2010.04.004.

[4] D.A. Knight, S.T. Holgate, The airway epithelium: structural and functional properties in health and disease, Respirology. 8 (2003) 432-446.

[5] A. Tam, S. Wadsworth, D. Dorscheid, S.F.P. Man, D.D. Sin, The airway epithelium: more than just a structural barrier, Ther Adv Respir Dis. 5 (2011) 255-273. https://doi.org/10.1177/1753465810396539.

[6] J.P. Soleas, A. Paz, P. Marcus, A. McGuigan, T.K. Waddell, Engineering airway epithelium, J. Biomed. Biotechnol. 2012 (2012) 982971. https://doi.org/10.1155/2012/982971.

[7] S.A. Shoyele, A. Slowey, Prospects of formulating proteins/peptides as aerosols for pulmonary drug 
delivery, Int J Pharm. 314 (2006) 1-8. https://doi.org/10.1016/j.ijpharm.2006.02.014.

[8] R. Belsey, Resection and reconstruction of the intrathoracic trachea, BJS (British Journal of Surgery). 38 (1950) 200-205. https://doi.org/10.1002/bjs.18003815008.

[9] P. Delaere, D. Van Raemdonck, Tracheal replacement, J Thorac Dis. 8 (2016) S186-S196. https://doi.org/10.3978/j.issn.2072-1439.2016.01.85.

[10] S. Ganesan, A.T. Comstock, U.S. Sajjan, Barrier function of airway tract epithelium, Tissue Barriers. 1 (2013) e24997. https://doi.org/10.4161/tisb.24997.

[11] C. Pfenninger, I. Leinhase, M. Endres, N. Rotter, A. Loch, J. Ringe, M. Sittinger, Tracheal remodeling: comparison of different composite cultures consisting of human respiratory epithelial cells and human chondrocytes, In Vitro Cell. Dev. Biol. Anim. 43 (2007) 28-36. https://doi.org/10.1007/s11626-006-9000-6.

[12] P.R. Delaere, D. Van Raemdonck, The trachea: The first tissue-engineered organ?, J Thorac Cardiovasc. 147 (2014) 1128-1132. https://doi.org/10.1016/j.jtcvs.2013.12.024.

[13] C. Johnson, P. Sheshadri, J.M. Ketchum, L.K. Narayanan, P.M. Weinberger, R.A. Shirwaiker, In vitro characterization of design and compressive properties of 3D-biofabricated/decellularized hybrid grafts for tracheal tissue engineering, J Mech Behav Biomed Mater. 59 (2016) 572-585. https://doi.org/10.1016/j.jmbbm.2016.03.024.

[14] S.J. Hollister, M.P. Hollister, S.K. Hollister, Computational modeling of airway instability and collapse in tracheomalacia, Respir Res. 18 (2017). https://doi.org/10.1186/s12931-017-0540-y.

[15] N.T. Remlinger, C.A. Czajka, M.E. Juhas, D.A. Vorp, D.B. Stolz, S.F. Badylak, S. Gilbert, T.W. Gilbert, Hydrated xenogeneic decellularized tracheal matrix as a scaffold for tracheal reconstruction, Biomaterials. 31 (2010) 3520-3526. https://doi.org/10.1016/j.biomaterials.2010.01.067.

[16] A. Batioglu-Karaaltin, M.V. Karaaltin, E. Ovali, O. Yigit, M. Kongur, O. Inan, E. Bozkurt, H. Cansiz, In vivo tissue-engineered allogenic trachea transplantation in rabbits: a preliminary report, Stem Cell Rev Rep. 11 (2015) 347-356. https://doi.org/10.1007/s12015-014-9570-8.

[17] A. Firth, J.X.-J. Yuan, eds., Lung stem cells in the epithelium and vasculature, Springer International Publishing, 2015. //www.springer.com/gp/book/9783319162317 (accessed September 4, 2018).

[18] Q. Tan, R. Steiner, S.P. Hoerstrup, W. Weder, Tissue-engineered trachea: History, problems and the future, Eur J Cardiothorac Surg. 30 (2006) 782-786. https://doi.org/10.1016/j.ejcts.2006.08.023.

[19] A. Tani, Y. Tada, T. Takezawa, I. Wada, M. Imaizumi, Y. Nomoto, M. Nomoto, K. Omori, Regenerative process of tracheal epithelium using a collagen vitrigel sponge scaffold, Laryngoscope. 123 (2013) 1469-1473. https://doi.org/10.1002/lary.23742.

[20] Y. Nomoto, K. Kobayashi, Y. Tada, I. Wada, T. Nakamura, K. Omori, Effect of fibroblasts on epithelial regeneration on the surface of a bioengineered trachea, Ann. Otol. Rhinol. Laryngol. 117 (2008) 59-64. https://doi.org/10.1177/000348940811700112.

[21] C. O'Leary, B. Cavanagh, R.E. Unger, C.J. Kirkpatrick, S. O’Dea, F.J. O’Brien, S.-A. Cryan, The development of a tissue-engineered tracheobronchial epithelial model using a bilayered collagenhyaluronate scaffold, Biomaterials. 85 (2016) 111-127. https://doi.org/10.1016/j.biomaterials.2016.01.065.

[22] J. An, J.E.M. Teoh, R. Suntornnond, C.K. Chua, Design and 3D printing of scaffolds and tissues, Engineering. 1 (2015) 261-268. https://doi.org/10.15302/J-ENG-2015061.

[23] S. Van Bael, Y.C. Chai, S. Truscello, M. Moesen, G. Kerckhofs, H. Van Oosterwyck, J.-P. Kruth, J. Schrooten, The effect of pore geometry on the in vitro biological behavior of human periosteumderived cells seeded on selective laser-melted Ti6Al4V bone scaffolds, Acta Biomater. 8 (2012) 2824-2834. https://doi.org/10.1016/j.actbio.2012.04.001. 
[24] L. Wu, J. Virdee, E. Maughan, A. Darbyshire, G. Jell, M. Loizidou, M. Emberton, P. Butler, A. Howkins, A. Reynolds, I.W. Boyd, M. Birchall, W. Song, Stiffness memory nanohybrid scaffolds generated by indirect 3D printing for biologically responsive soft implants, Acta Biomater. 80 (2018) 188-202. https://doi.org/10.1016/j.actbio.2018.09.016.

[25] L. Wu, A. Magaz, T. Wang, C. Liu, A. Darbyshire, M. Loizidou, M. Emberton, M. Birchall, W. Song, Stiffness memory of indirectly 3D-printed elastomer nanohybrid regulates chondrogenesis and osteogenesis of human mesenchymal stem cells, Biomaterials. 186 (2018) 64-79. https://doi.org/10.1016/j.biomaterials.2018.09.013.

[26] L. Wu, A. Magaz, A. Darbyshire, A. Howkins, A. Reynolds, I.W. Boyd, H. Song, J.-H. Song, M. Loizidou, M. Emberton, M. Birchall, W. Song, Thermoresponsive stiffness softening of hierarchically porous nanohybrid membranes promotes niches for mesenchymal stem cell differentiation, Adv Healthc Mater. 8 (2019) 1801556. https://doi.org/10.1002/adhm.201801556.

[27] L. Wu, A. Magaz, E. Maughan, N. Oliver, A. Darbyshire, M. Loizidou, M. Emberton, M. Birchall, W. Song, Cellular responses to thermoresponsive stiffness memory elastomer nanohybrid scaffolds by 3D-TIPS, Acta Biomater. 85 (2019) 157-171. https://doi.org/10.1016/j.actbio.2018.12.019.

[28] I. Massie, A.K. Kureshi, S. Schrader, A.J. Shortt, J.T. Daniels, Optimization of optical and mechanical properties of real architecture for 3-dimensional tissue equivalents: Towards treatment of limbal epithelial stem cell deficiency, Acta Biomater. 24 (2015) 241-250. https://doi.org/10.1016/j.actbio.2015.06.007.

[29] E. Melo, J.Y. Kasper, R.E. Unger, R. Farré, C.J. Kirkpatríck, Development of a bronchial wall model: triple culture on a decellularized porcine trachea, Tissue Eng Part C-ME. 21 (2015) 909921. https://doi.org/10.1089/ten.tec.2014.0543.

[30] C.I. Grainger, L.L. Greenwell, D.J. Lockley, G.P. Martin, B. Forbes, Culture of Calu-3 cells at the air interface provides a representative model of the airway epithelial barrier, Pharm Res. 23 (2006) 1482-1490. https://doi.org/10.1007/s11095-006-0255-0.

[31] E.M. Renkin, Filtration, diffusion, and molecular sieving through porous cellulose membranes, J. Gen. Physiol. 38 (1954) 225-243.

[32] A. Batioglu-Karaaltin, E. Ovali, M.V. Karaaltin, M. Yener, M. Yılmaz, F. Eyüpoğlu, Y.Z. Yılmaz, E.R. Bozkurt, N. Demir, E. Konuk, E.S. Bozdağ, Ö. Yiğit, H. Cansiz, Decellularization of trachea with combined techniques for tissue-engineered trachea transplantation, Clin Exp Otorhinolaryngol. 12 (2018) 86-94. https://doi.org/10.21053/ceo.2018.00486.

[33] J.J. Urbano, R.K. da Palma, F.M. de Lima, P. Fratini, L.L. Guimaraes, J.J. Uriarte, L.H. Alvarenga, M.A. Miglino, R. de P. Vieira, R.A. Prates, D. Navajas, R. Farrè, L.V.F. Oliveira, Effects of two different decellularization routes on the mechanical properties of decellularized lungs, PLOS ONE. 12 (2017) e0178696. https://doi.org/10.1371/journal.pone.0178696.

[34] K. Omori, Y. Tada, T. Suzuki, Y. Nomoto, T. Matsuzuka, K. Kobayashi, T. Nakamura, S. Kanemaru, M. Yamashita, R. Asato, Clinical application of in situ tissue engineering using a scaffolding technique for reconstruction of the larynx and trachea, Ann. Otol. Rhinol. Laryngol. 117 (2008) 673-678. https://doi.org/10.1177/000348940811700908.

[35] K. Lefort, G.P. Dotto, p63 and epithelial metaplasia: a gutsy choice, Cell. 145 (2011) 1003-1005. https://doi.org/10.1016/j.cell.2011.06.008.

[36] J.K. Rains, J.L. Bert, C.R. Roberts, P.D. Paré, Mechanical properties of human tracheal cartilage, J. Appl. Physiol. 72 (1992) 219-225. https://doi.org/10.1152/jappl.1992.72.1.219.

[37] F. Safshekan, M. Tafazzoli-Shadpour, M. Abdouss, M.B. Shadmehr, Mechanical characterization and constitutive modeling of human trachea: age and gender dependency, Materials. 9 (2016) 456. https://doi.org/10.3390/ma9060456.

[38] C.R. Butler, R.E. Hynds, C. Crowley, K.H.C. Gowers, L. Partington, N.J. Hamilton, C. Carvalho, 
M. Platé, E.R. Samuel, A.J. Burns, L. Urbani, M.A. Birchall, M.W. Lowdell, P. De Coppi, S.M. Janes, Vacuum-assisted decellularization: an accelerated protocol to generate tissue-engineered human tracheal scaffolds, Biomaterials. 124 (2017) 95-105. https://doi.org/10.1016/j.biomaterials.2017.02.001.

[39] J.-Y. Wang, P. Mesquida, T. Lee, Young's modulus measurement on pig trachea and bronchial airways, Conf Proc IEEE Eng Med Biol Soc. 2011 (2011) 2089-2092. https://doi.org/10.1109/IEMBS.2011.6090388.

[40] B. Hoffman, M. Martin, B.N. Brown, L.J. Bonassar, J. Cheetham, Biomechanical and biochemical characterization of porcine tracheal cartilage, Laryngoscope. 126 (2016) E325-331. https://doi.org/10.1002/lary.25861.

[41] M.P.E. Wenger, L. Bozec, M.A. Horton, P. Mesquida, Mechanical properties of collagen fibrils, Biophys J. 93 (2007) 1255-1263. https://doi.org/10.1529/biophysj.106.103192.

[42] C. Kayal, R.J. Shipley, J.B. Phillips, Physical and mechanical properties of RAFT-stabilised collagen gels for tissue engineering applications, J Mech Behav Biomed Mater. 99 (2019) 216-224. https://doi.org/10.1016/j.jmbbm.2019.07.011.

[43] M. Zang, Q. Zhang, E.I. Chang, A.B. Mathur, P. Yu, Decellularized tracheal matrix scaffold for tissue engineering, Plast. Reconstr. Surg. 130 (2012) 532-540. https://doi.org/10.1097/PRS.0b013e31825dc084.

[44] K. Kobayashi, T. Suzuki, Y. Nomoto, Y. Tada, M. Miyake, A. Hazama, I. Wada, T. Nakamura, K. Omori, A tissue-engineered trachea derived from a framed collagen scaffold, gingival fibroblasts and adipose-derived stem cells, Biomaterials. 31 (2010) 4855-4863. https://doi.org/10.1016/j.biomaterials.2010.02.027.

[45] S.L. Thibeault, S.A. Klemuk, X. Chen, B.H. Quinchia Johnson, In vivo engineering of the vocal fold ECM with injectable ha hydrogels -- late effects on tissue repair and biomechanics in a rabbit model, J Voice. 25 (2011) 249-253. https://doi.org/10.1016/j.jvoice.2009.10.003.

[46] S.P. Choksi, G. Lauter, P. Swoboda, S. Roy, Switching on cilia: transcriptional networks regulating ciliogenesis, Development. 141 (2014) 1427-1441. https://doi.org/10.1242/dev.074666.

[47] C. Pohl, M.I. Hermanns, C. Uboldi, M. Bock, S. Fuchs, J. Dei-Anang, E. Mayer, K. Kehe, W. Kummer, C.J. Kirkpatrick, Barrier functions and paracellular integrity in human cell culture models of the proximal respiratory unit, Eur J Pharm Biopharm. 72 (2009) 339-349. https://doi.org/10.1016/j.ejpb.2008.07.012.

[48] P.L. Leopold, M.J. O'Mahony, X.J. Lian, A.E. Tilley, B.-G. Harvey, R.G. Crystal, Smoking is associated with shortened airway cilia, PLOS ONE. 4 (2009) e8157. https://doi.org/10.1371/journal.pone.0008157.

[49] C. O'Leary, L. Soriano, A. Fagan-Murphy, I. Ivankovic, B. Cavanagh, F.J. O’Brien, S.-A. Cryan, The Fabrication and in vitro Evaluation of Retinoic Acid-Loaded Electrospun Composite Biomaterials for Tracheal Tissue Regeneration, Front. Bioeng. Biotechnol. 8 (2020). https://doi.org/10.3389/fbioe.2020.00190.

[50] C. O'Leary, F.J. O’Brien, S.-A. Cryan, Retinoic Acid-Loaded Collagen-Hyaluronate Scaffolds: A Bioactive Material for Respiratory Tissue Regeneration, ACS Biomater. Sci. Eng. 3 (2017) 13811393. https://doi.org/10.1021/acsbiomaterials.6b00561.

[51] M.S. Ali, J.P. Pearson, Upper airway mucin gene expression: a review, The Laryngoscope. 117 (2007) 932-938. https://doi.org/10.1097/MLG.0b013e3180383651.

[52] D.J. Thornton, K. Rousseau, M.A. McGuckin, Structure and function of the polymeric mucins in airways mucus, Annu. Rev. Physiol. 70 (2008) 459-486. https://doi.org/10.1146/annurev.physiol.70.113006.100702.

[53] M.M. Choe, P.H.S. Sporn, M.A. Swartz, Extracellular matrix remodeling by dynamic strain in a 
three-dimensional tissue-engineered human airway wall model, Am. J. Respir. Cell Mol. Biol. 35 (2006) 306-313. https://doi.org/10.1165/rcmb.2005-0443OC.

[54] B.S. Schuster, J.S. Suk, G.F. Woodworth, J. Hanes, Nanoparticle diffusion in respiratory mucus from humans without lung disease, Biomaterials. 34 (2013) 3439-3446. https://doi.org/10.1016/j.biomaterials.2013.01.064.

[55] A. Hartsock, W.J. Nelson, Adherens and tight junctions: structure, function and connections to the actin cytoskeleton, Biochim Biophys Acta. 1778 (2008) 660-669. https://doi.org/10.1016/j.bbamem.2007.07.012.

[56] A.I. Ivanov, C.A. Parkos, A. Nusrat, Cytoskeletal regulation of epithelial barrier function during inflammation, Am. J. Clin. Pathol. 177 (2010) 512-524. https://doi.org/10.2353/ajpath.2010.100168.

[57] J.A. Rhodin, The ciliated cell. Ultrastructure and function of the human tracheal mucosa, Am. Rev. Respir. Dis. 93 (1966) Suppl:1-15. https://doi.org/10.1164/arrd.1966.93.3P2.1.

[58] X.M. Bustamante-Marin, L.E. Ostrowski, Cilia and mucociliary clearance, Cold Spring Harb Perspect Biol. 9 (2017). https://doi.org/10.1101/cshperspect.a028241.

[59] B. Forbes, C. Ehrhardt, Human respiratory epithelial cell culture for drug delivery applications, Eur J Pharm Biopharm. 60 (2005) 193-205. https://doi.org/10.1016/j.ejpb.2005.02.010.

[60] R. Derk, D.C. Davidson, A. Manke, T.A. Stueckle, Y. Rojanasakul, L. Wang, Potential in vitro model for testing the effect of exposure to nanoparticles on the lung alveolar epithelial barrier, Sensing and Bio-Sensing Research. 3 (2015) 38-45. https://doi.org/10.1016/j.sbsr.2014.12.002.

[61] S. Tian, W. Ren, G. Li, R. Yang, T. Wang, A theoretical analysis of pore size distribution effects on shale apparent permeability, Geofluids. (2017). https://doi.org/10.1155/2017/7492328.

[62] N. Zhong, T. Dong, Z. Chen, Y. Guo, Z. Shao, X. Zhao, A novel 3D-printed silk fibroin-based scaffold facilitates tracheal epithelium proliferation in vitro, J Biomater Appl. 34 (2019) 3-11. https://doi.org/10.1177/0885328219845092.

[63] O.A. Romanova, T.H. Tenchurin, T.S. Demina, E.V. Sytina, A.D. Shepelev, S.G. Rudyak, O.I. Klein, S.V. Krasheninnikov, E.I. Safronova, R.A. Kamyshinsky, V.G. Mamagulashvili, T.A. Akopova, S.N. Chvalun, A.A. Panteleyev, Non-woven bilayered biodegradable chitosan-gelatin-polylactide scaffold for bioengineering of tracheal epithelium, Cell Proliferation. 52 (2019) e12598. https://doi.org/10.1111/cpr.12598.

[64] S.J. Hollister, C.L. Flanagan, D.A. Zopf, R.J. Morrison, H. Nasser, J.J. Patel, E. Ebramzadeh, S.N. Sangiorgio, M.B. Wheeler, G.E. Green, Design control for clinical translation of 3D printed modular scaffolds, Ann Biomed Eng. 43 (2015) 774-786. https://doi.org/10.1007/s10439-015-1270-2. 


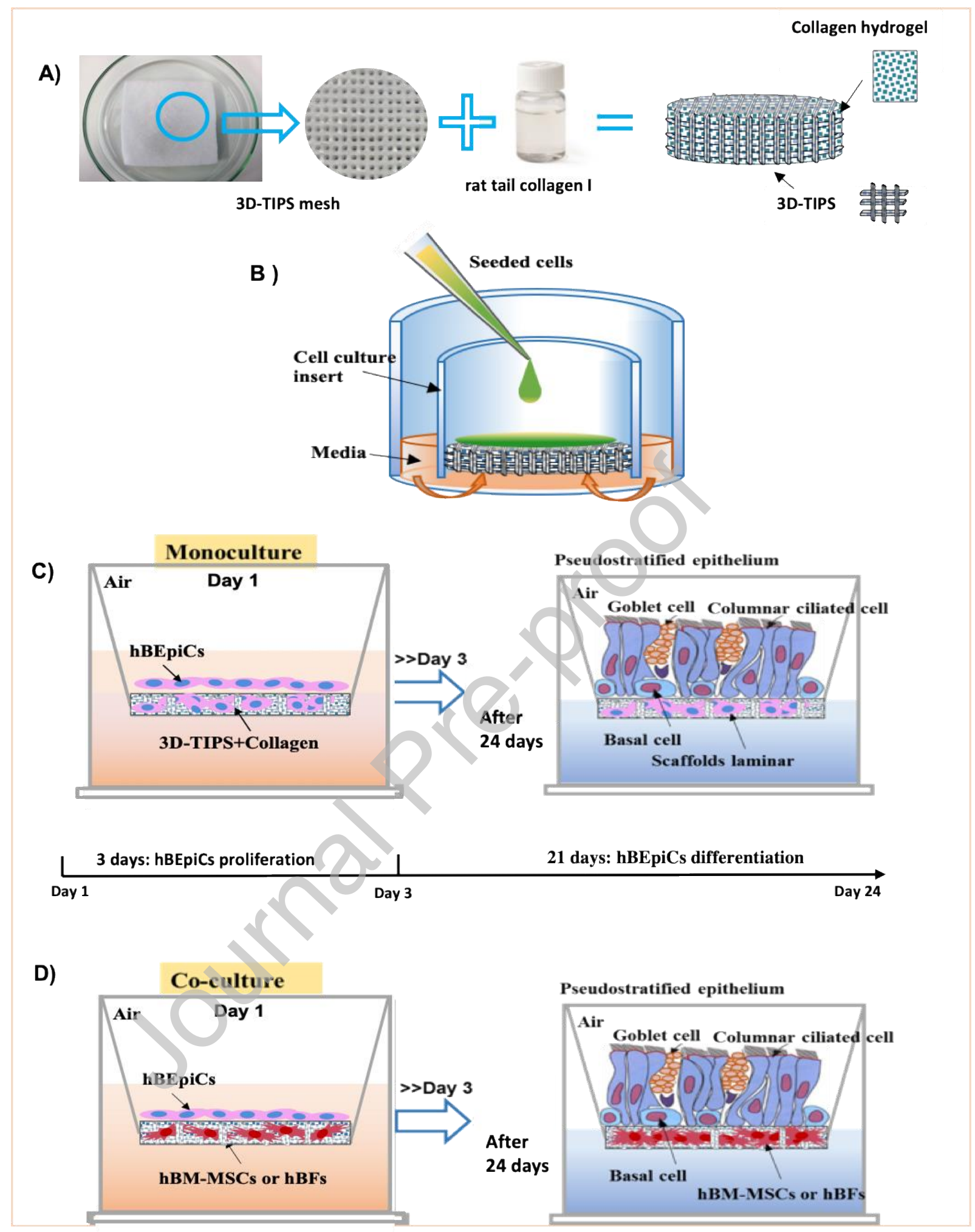

Scheme 1 (A) Schematic of collagen hydrogel functionalized 3D-TIPS elastomer scaffolds (3D-

TIPS+Collagen). (B-D) Illustration of air liquid interface cell culture of human bronchial epithelium cells (hBEpiCs) on the scaffolds. The cells are seeded on the scaffold and cultured in the medium to 
encourage cell attachment, migration and proliferation for 3 days, and the scaffolds are air-lifted subsequently and the cells are exposed to an air-liquid interface over 21 days: (C) hBEpiCs in monoculture conditions; and (D) hBEpiCs in co-culture conditions with either bone-marrow derived mesenchymal stem cells (hBM-MSCs) or human bronchial fibroblast cells (hBFs) underneath.
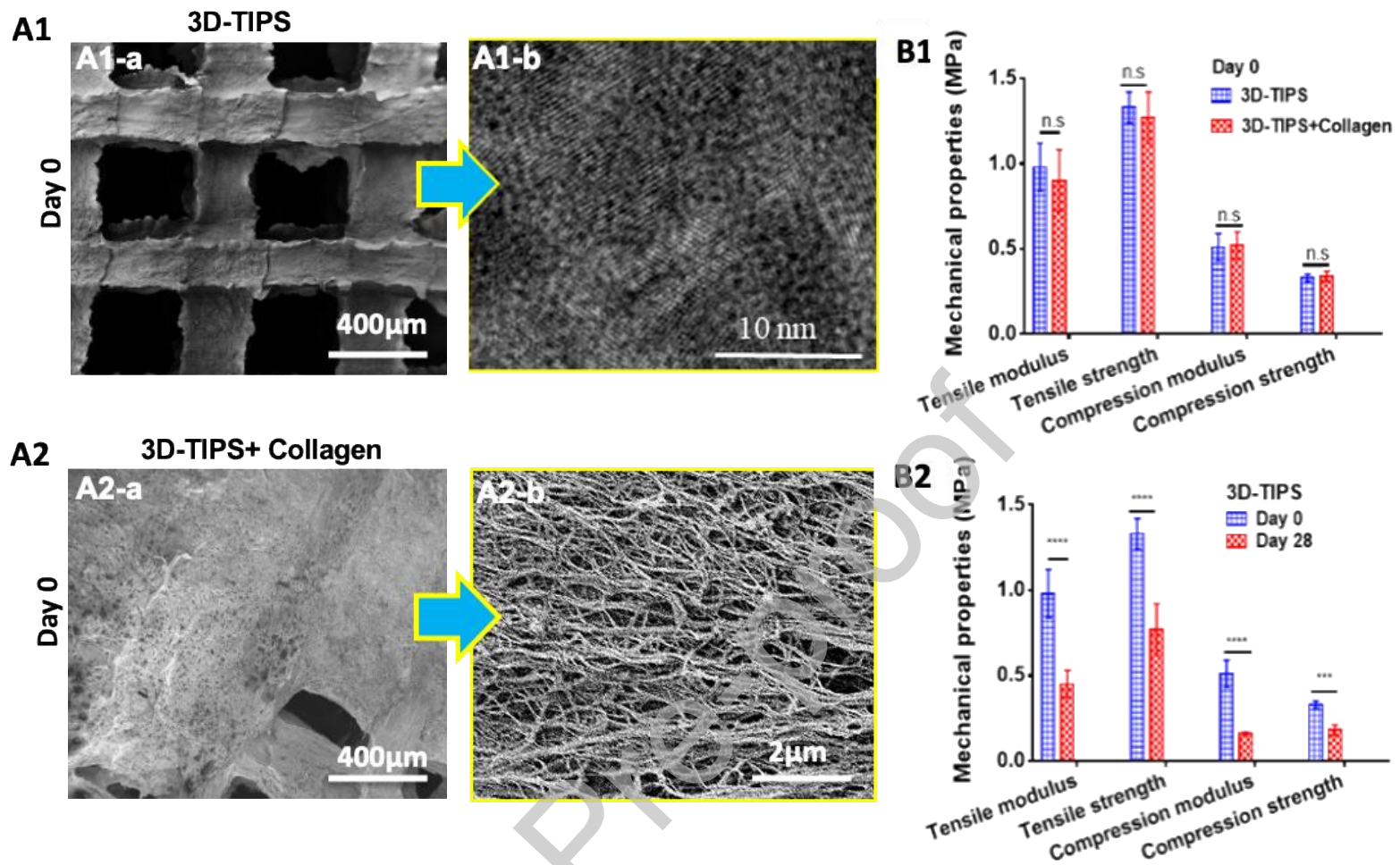

C1
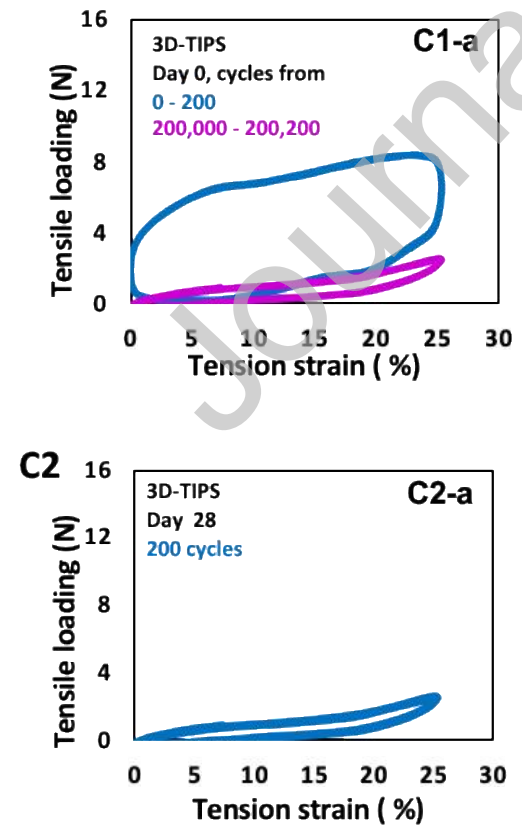
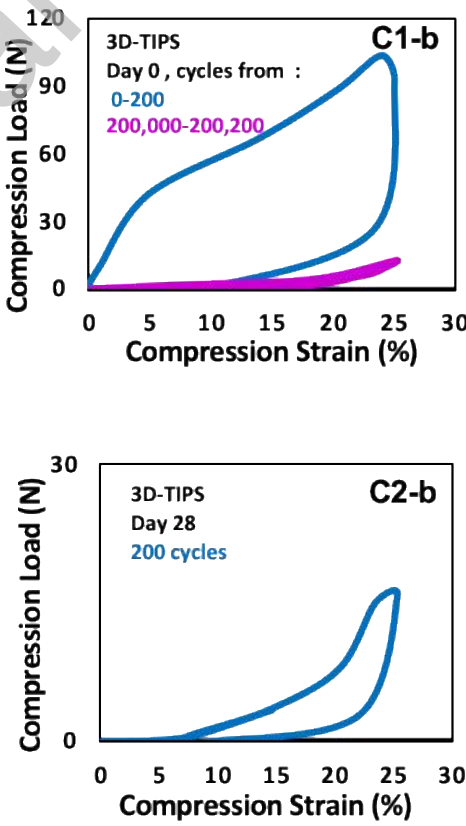

D1

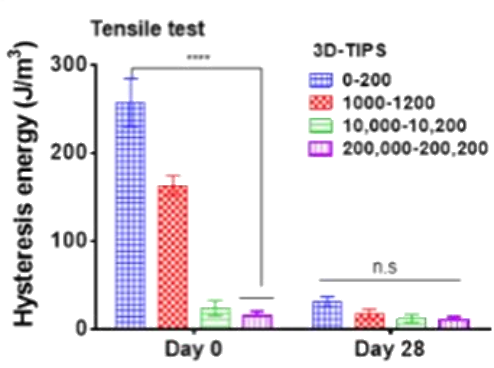

D2

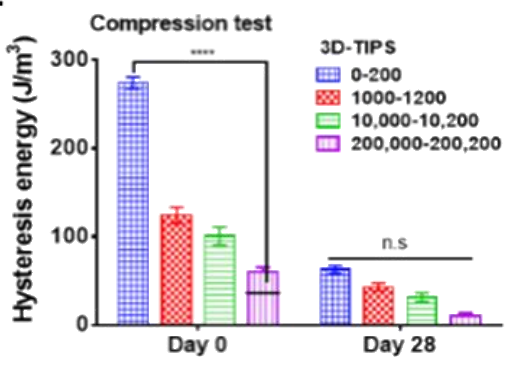


Fig. 1 (A1) Representative SEM micrograph of the top-surface of the 3D-TIPS scaffold (A1-a) and high resolution TEM image of the nano-phase structure (A1-b). (A2) SEM micrograph of the 3D-TIPS+Collagen scaffold at two different magnifications. (B-D) Static and dynamic mechanical properties of the scaffolds ( $n=6$ per type): (B1) comparison of the static tensile and compressive properties of 3D-TIPS and 3DTIPS+Collagen scaffolds as produced at day 0; (B2) stiffness softening of 3D-TIPS after 28 days incubation at body temperature, assessed in terms of static tensile and compressive properties; (C1) stiffness softening of 3D-TIPS as produced at day 0 in dynamic tensile (C1-a) and compression (C1-b) modes, accelerated at increasing cyclic loadings up to 200200 cycles; (C2) stiffness softening of 3D-TIPS in dynamic tensile (C2-a) and compression (C2-b) modes at 200 cycles after 28 days incubation; (D1-D2) hysteresis energy of 3D-TIPS assessed in terms of dynamic tensile and compressive cyclic loadings. Differences between the groups were analyzed by two-way ANOVA using Tukey's post hoc test. n.s nonsignificant; ${ }^{* * *} p<0.001 ;{ }^{* * * *} p<0.0001$. 
A

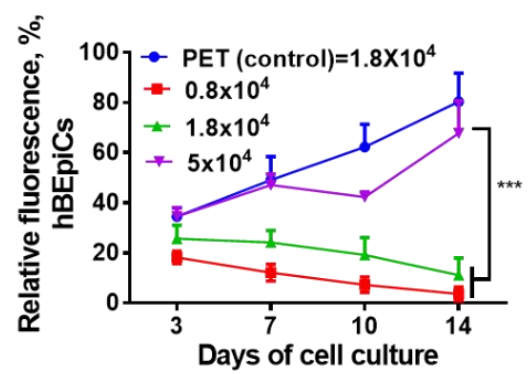

$\square$ hBEpiCs PET $\boxplus$ hBEpiCs 3D-TIPS

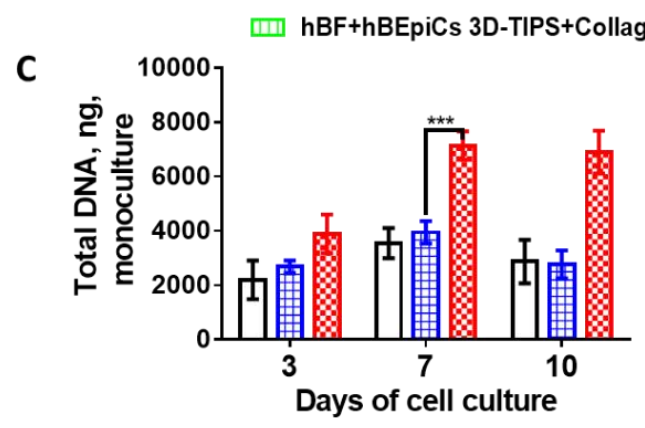

hBEpiCs 3D-TIPS
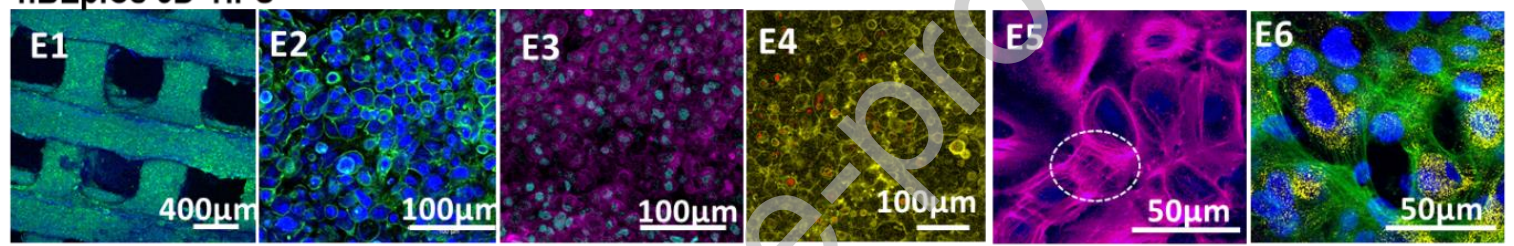

\section{hBEpiCs 3D-TIPS+Collagen}
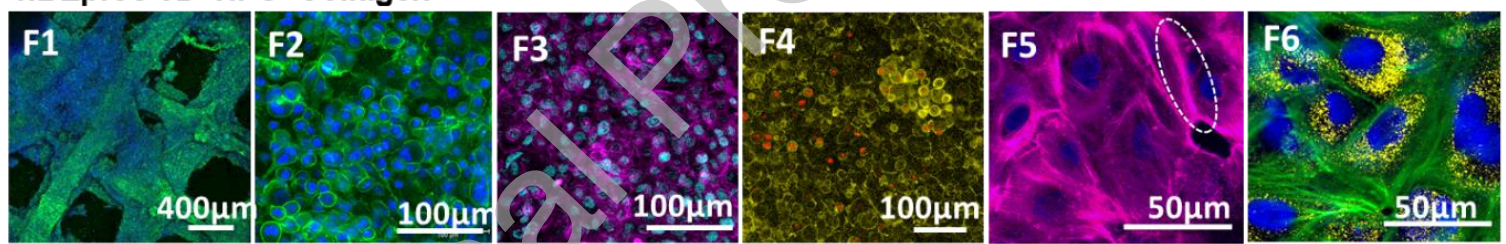

hBF+hBEpiCs 3D-TIPS+Collagen
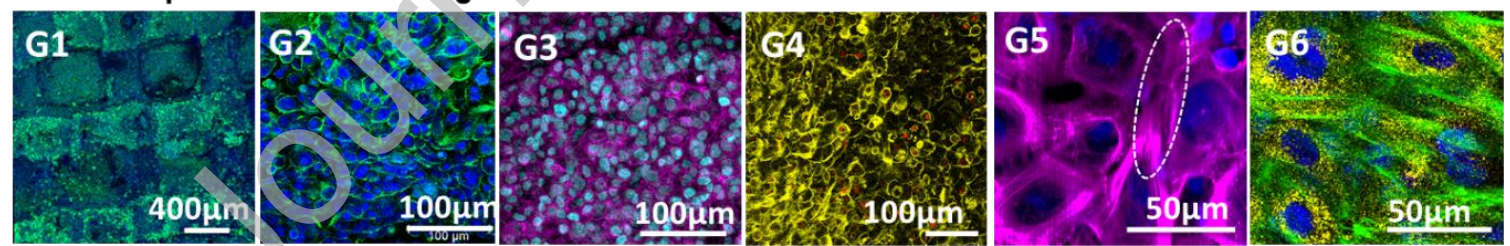

\section{hBM-MSCs+hBEpiCs 3D-TIPS+Collagen}
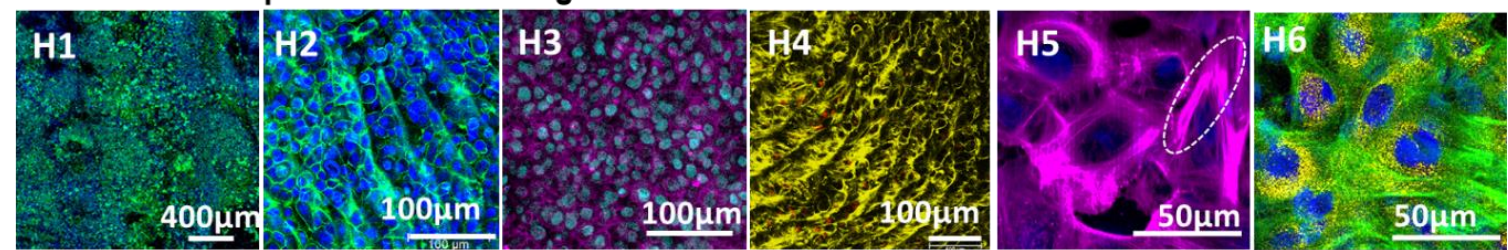

- Nuclei

F-actin $\square$ p63 $\square$ KRT 5

Ki67

Vimentin

Fig. 2 (A) Cellular viability of hBEpiCs seeded on 3D-TIPS scaffolds ( $n=6$ per type) with different cell seeding densities, measured by alamarBlue for 14 days. (B) AlamarBlue fluorescence assay ( $n=6$ per 
scaffold type) and (C) total DNA analysis ( $n=6$ per scaffold type) of hBEpiCs monocultured on 3D-TIPS and 3D-TIPS+Collagen scaffolds. (D) Percentage cell count of stained cells on the various scaffolds $(n=2$ per type; 10 frames per scaffold with 500 cells analyzed). (E-H) Representative confocal microscope images at day 7 showing hBEpiCs proliferation in each condition ( $n=2$ per scaffold type): (E) hBEpiCs monoculture on untreated 3D-TIPS; (F) hBEpiCs monoculture on 3D-TIPS+collagen; (G) hBEpiCs coculture with hBFs on 3D-TIPS+Collagen; (H) hBEpiCs co-culture with hBM-MSCs on 3D-TIPS+Collagen. Cells were stained respectively for nuclei (blue), f-actin (green), p63 (cyan), keratin 5 (magenta), Ki-67 expression (red) and vimentin (yellow). Differences between the groups were analyzed by two-way ANOVA using Tukey's post hoc test. n.s non-significant; ${ }^{* *} p<0.01 ;{ }^{* *} p<0.001$. 
h B E p IC s 3 D - T IP S

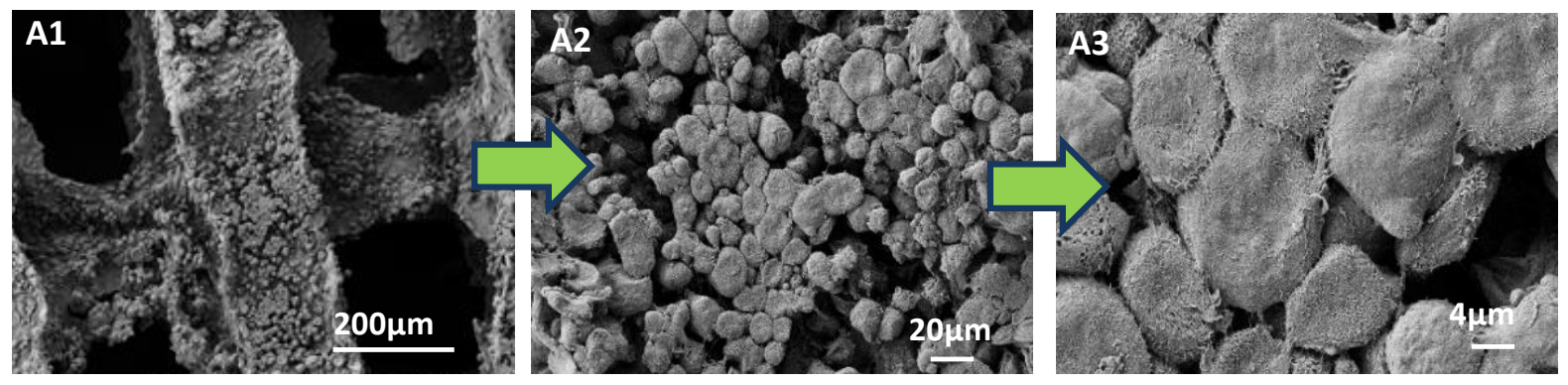

h B E piCs 3 D - T IP S + C ollag e n

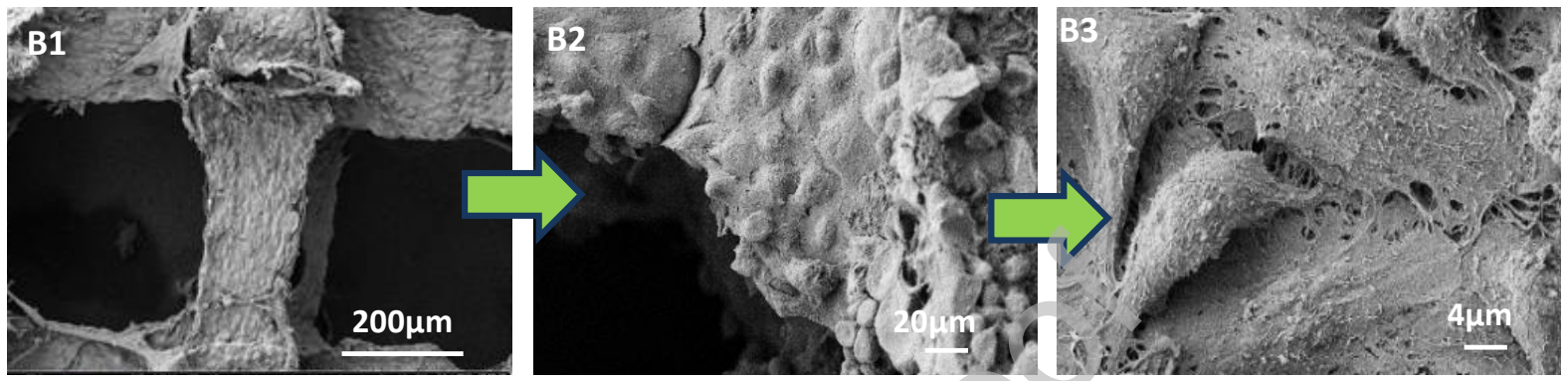

h B F + h B E p iCs 3 D -T IP S + C o Ilage n

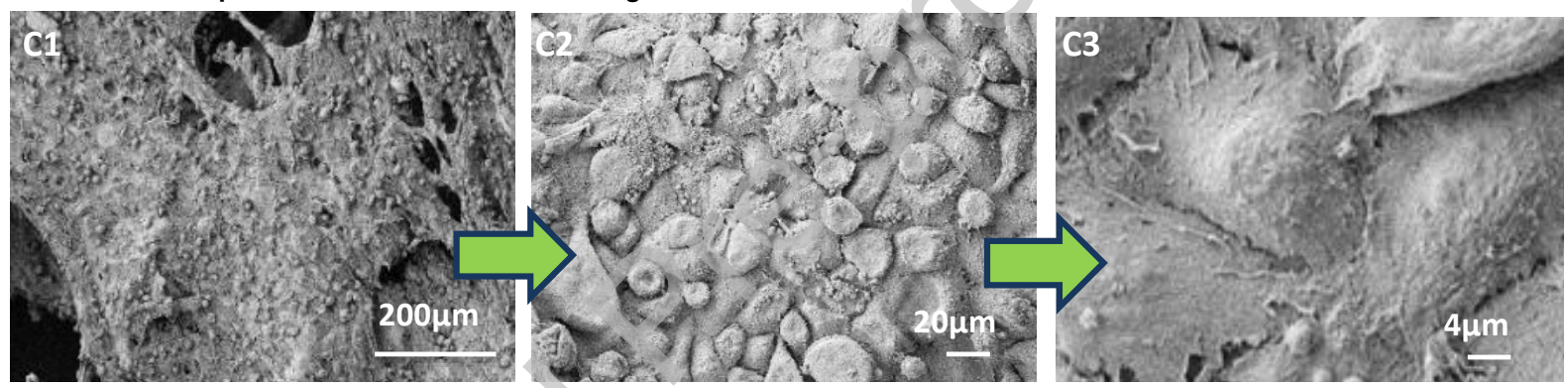

h B M - M S C s + h B E p iCs $3 D-T$ IP $S+C$ ollage n

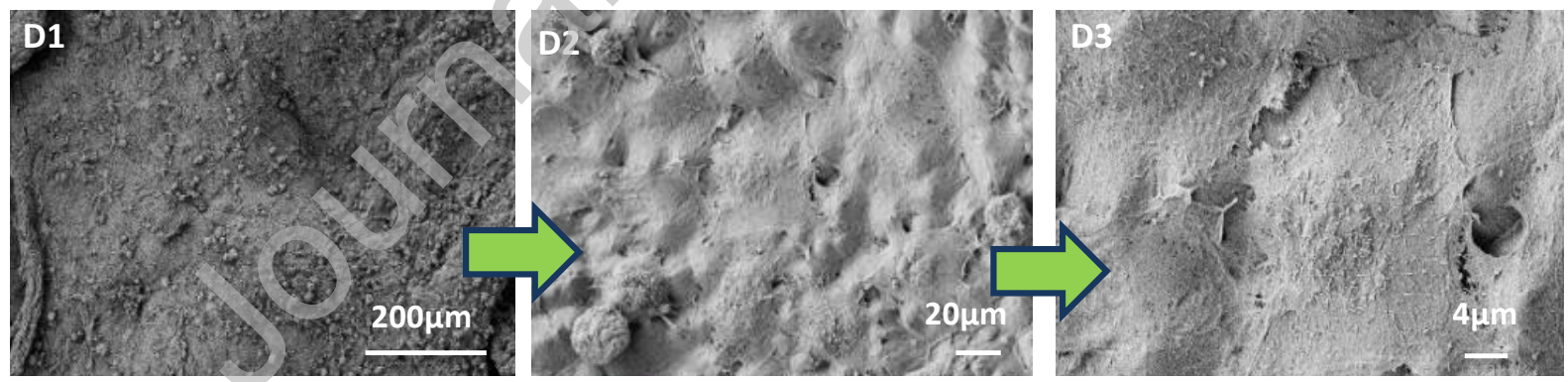

Fig. 3 Representative SEM micrographs showing hBEpiCs proliferation on each conditional scaffold at day 7 ( $n=3$ per type); (A) hBEpiCs monocultured on untreated 3D-TIPS; (B) hBEpiCs monocultured on 3DTIPS+Collagen; (C) hBEpiCs co-cultured with hBFs on 3D-TIPS+Collagen; (D) hBEpiCs co-cultured with hBM-MSCs on 3D-TIPS+Collagen. 

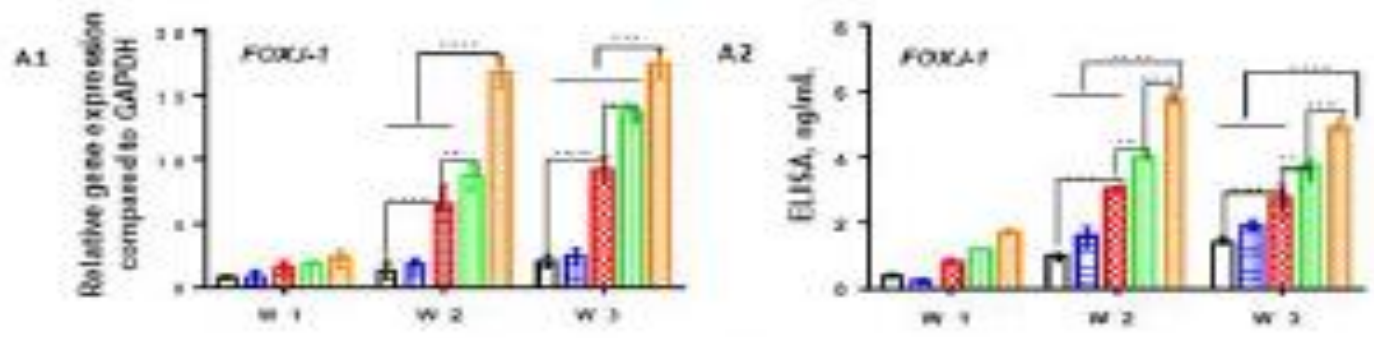

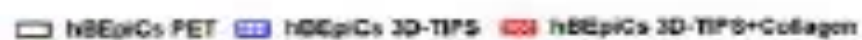

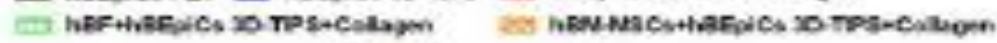
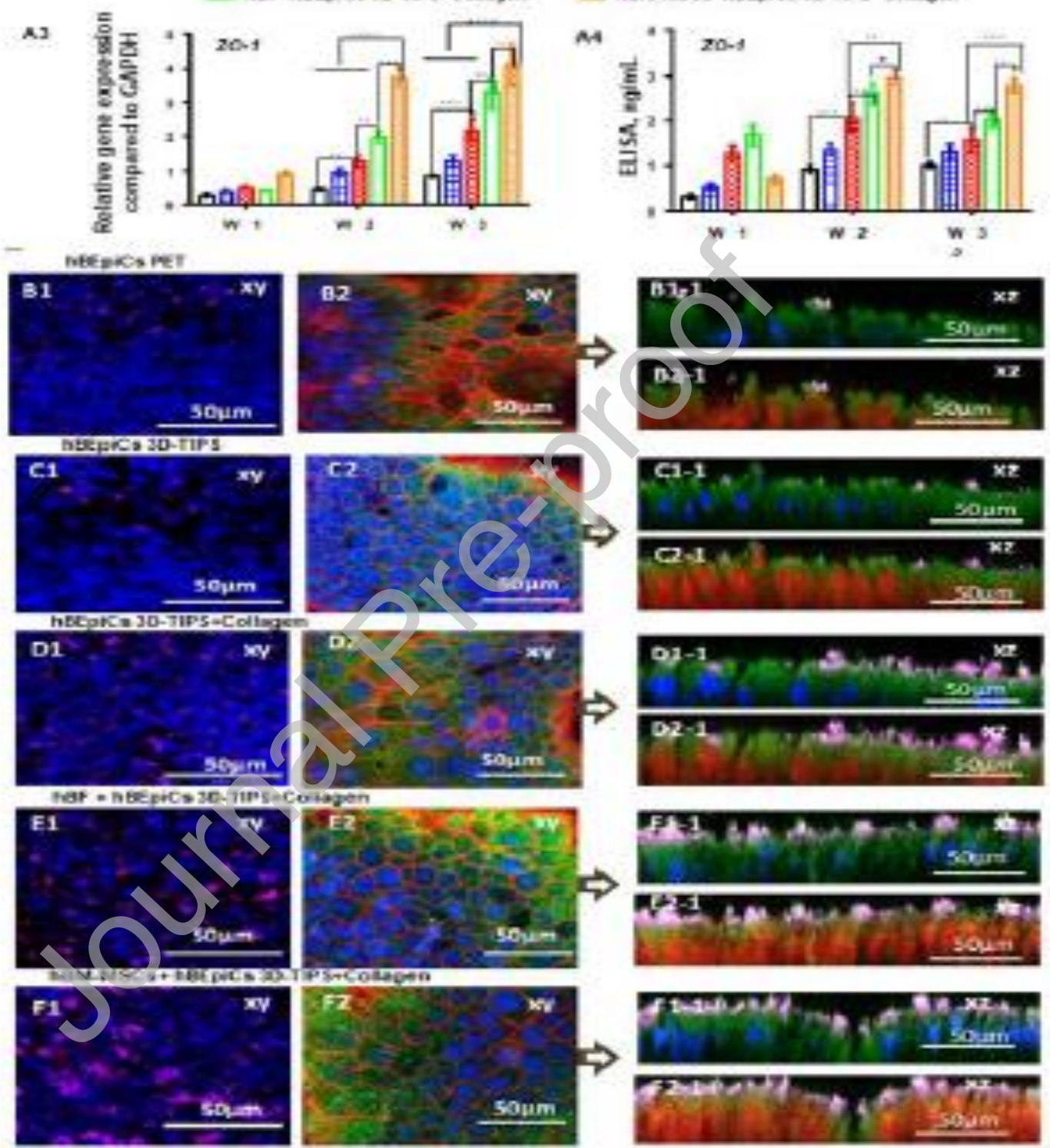

zO-1 Euclei $\mathbf{E}$ E-cadherin $\mathrm{E}$ acetylated a-ubulin

Fig. 4 (A1-A2) qPCR ( $n=5$ per scaffold type) and ELISA analysis ( $n=4$ per scaffold type) for FOXJ1 as a marker for ciliation; (A3-A4) qPCR ( $n=5$ per scaffold type) and ELISA analysis ( $n=4$ per scaffold type) for ZO-1 as a marker for tight junctions. (B-F) Representative confocal microscope images ( $n=2$ per scaffold 
type) at day 21 showing (B1-F1) acetylated alpha tubulin and (B2-F2) ZO-1 protein expression, respectively for ciliation and adherens junction for the various scaffolds: (B) hBEpiCs monoculture on PET cell culture insert; (C) hBEpiCs monoculture on untreated 3D-TIPS; (D) hBEpiCs monoculture on 3DTIPS+Collagen; (E) hBEpiCs co-culture with hBFs on 3D-TIPS+Collagen; (F) hBEpiCs co-culture with hBMMSCs on 3D-TIPS+Collagen. Maximum intensity projections of ZO-1 (red) reconstructed from Z-stacks; cells counterstained for nuclei (blue) and E-cadherin (green), with cilia indicated by acetylated alpha tubulin (purple). Differences between the groups were analyzed by two-way ANOVA using Tukey's post hoc test. ${ }^{* *} p<0.01 ;{ }^{* * *} p<0.001 ; * * * * p<0.0001$. 

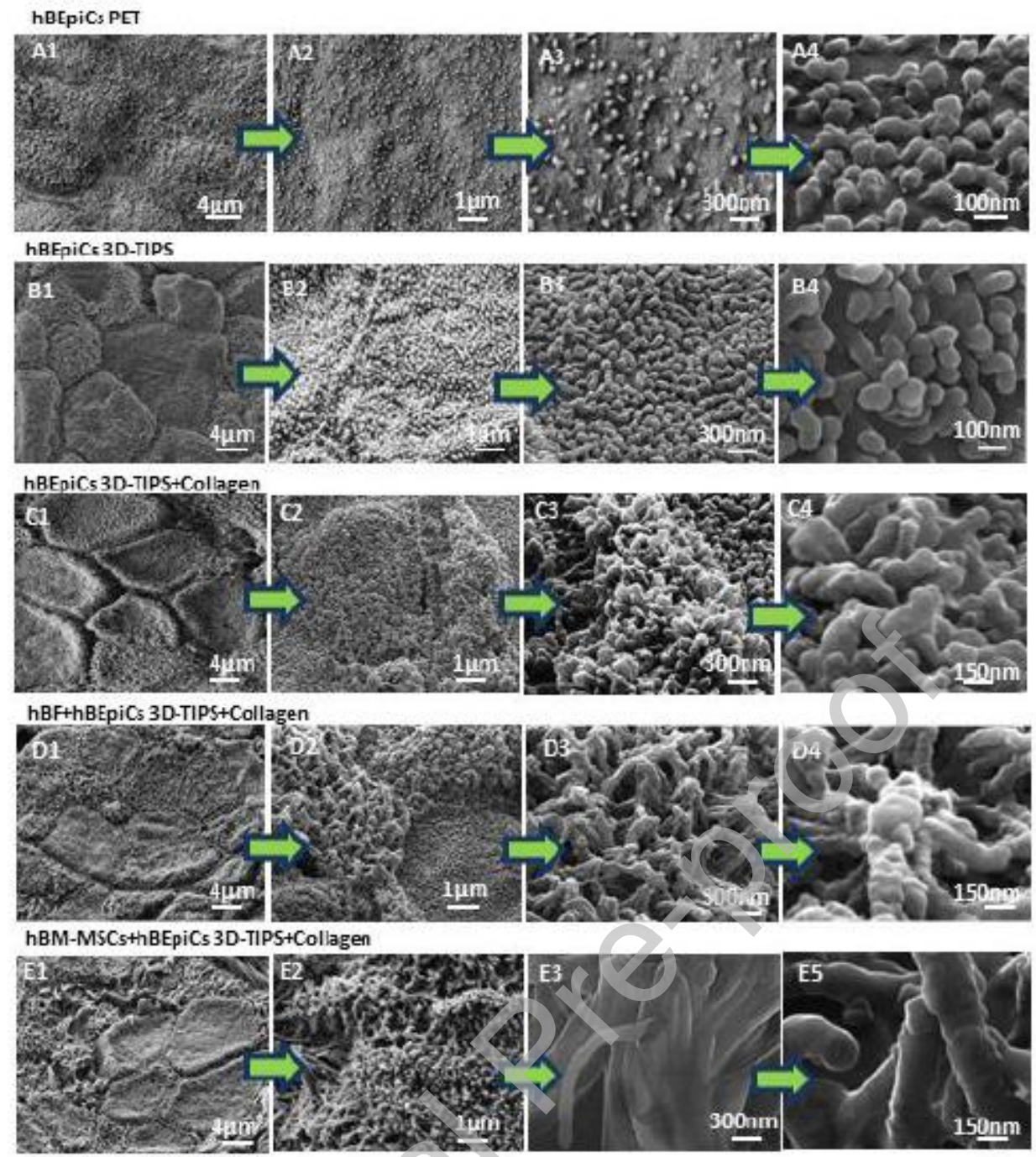

hBEpiCs 3D-TIPS+Collagen
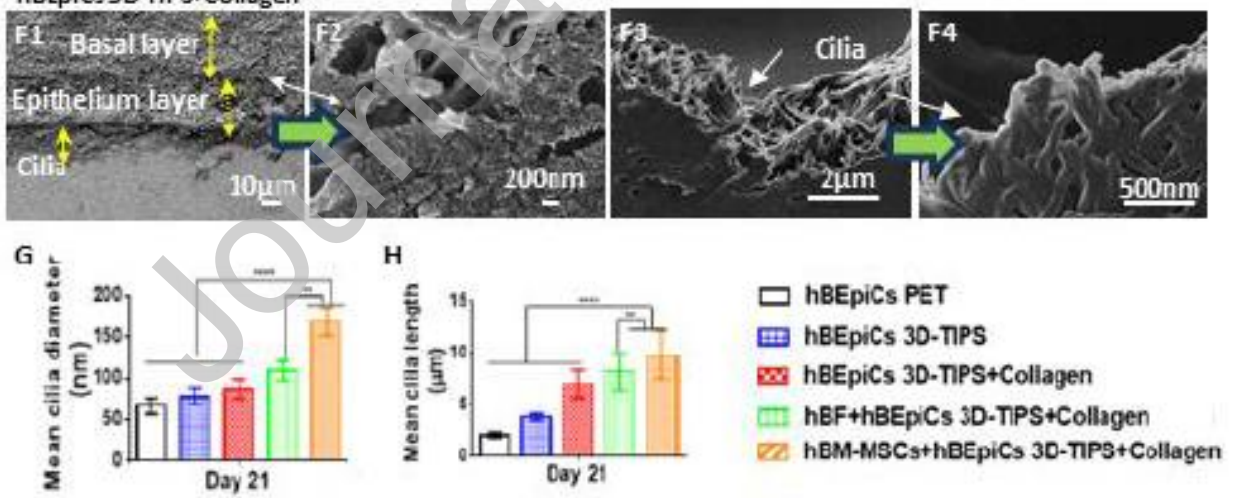

Fig. 5 (A-E) Representative He-ion FIB images of hBEpiCs differentiation in an air-liquid interface (ALI) culture on the various scaffolds ( $n=3$ per type) at day 21: (A) hBEpiCs monoculture on PET cell culture insert; (B) hBEpiCs monoculture on untreated 3D-TIPS; (C) hBEpiCs monoculture on 3D-TIPS+Collagen; (D) hBEpiCs co-culture with hBFs on 3D-TIPS+Collagen; (E) hBEpiCs co-culture with hBM-MSCs on 3DTIPS+Collagen. (F) Representative cross-sectional slices showing a ciliated epithelium of hBEpiCs 
monoculture on 3D-TIPS+Collagen scaffolds ( $n=3$ per type) at day 21. (G) Average mean cilia length and (H) average mean cilia diameter at day 21, evaluated from 6 images ( $n=20$ counts per image) at different fields of view within each scaffold ( $n=3$ per type). Differences between the groups were analyzed by one-way ANOVA using Tukey's post hoc test. ${ }^{* *} p<0.01 ; * * * * p<0.0001$. 
A1

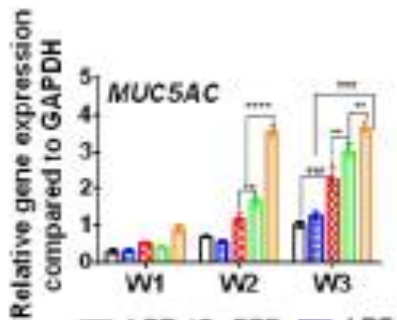

¿
A2

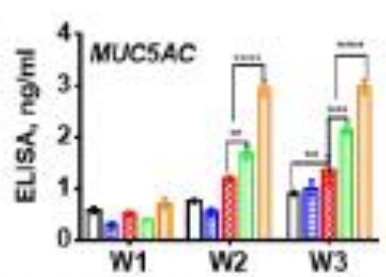

ZZZ hEM-MSCs+hBEpiCs 30-TIPS+Collagen

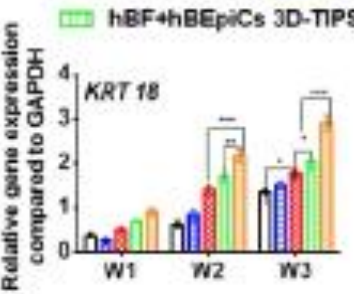

A4

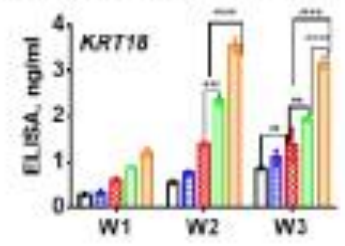

HBEpiCs PET
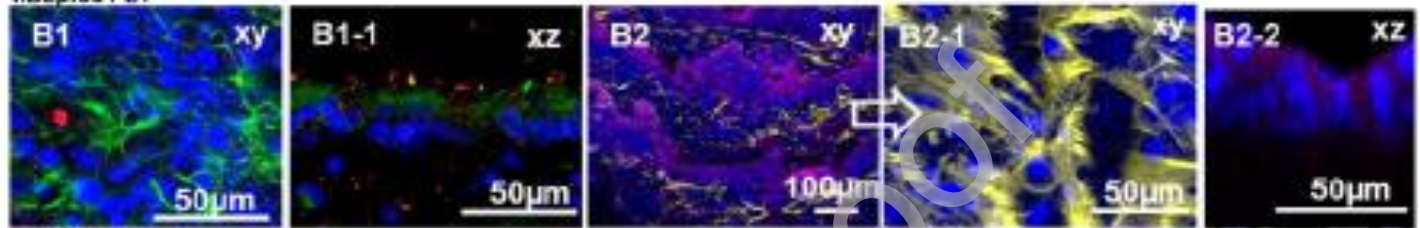

MBEDics SD.TPS

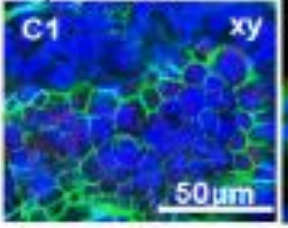

C1-1

$50 \mu \mathrm{m}$

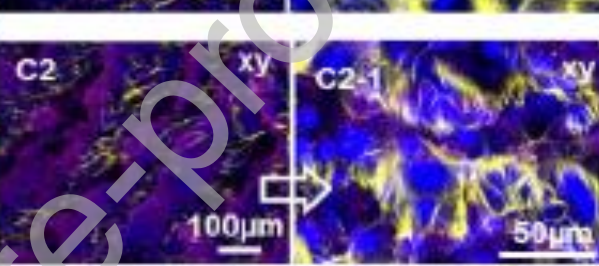

C2-2

$x z$
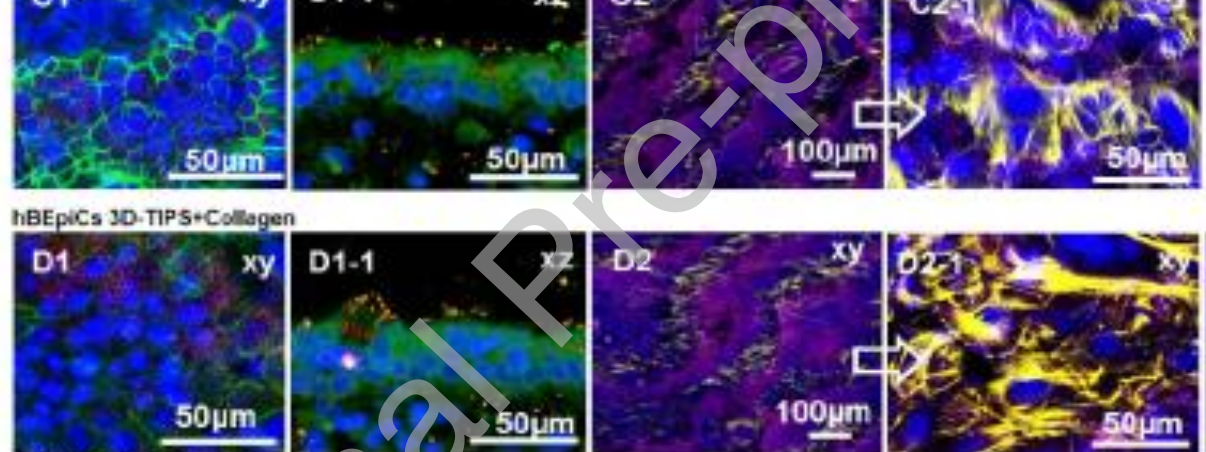

D2-2

$504 m$
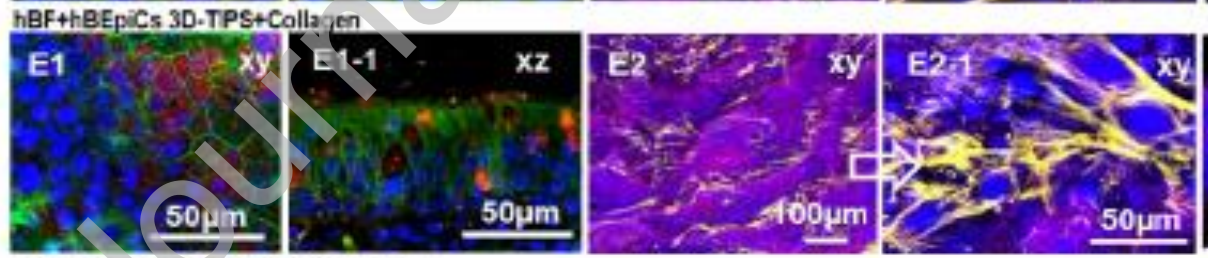

E2-2

50 um
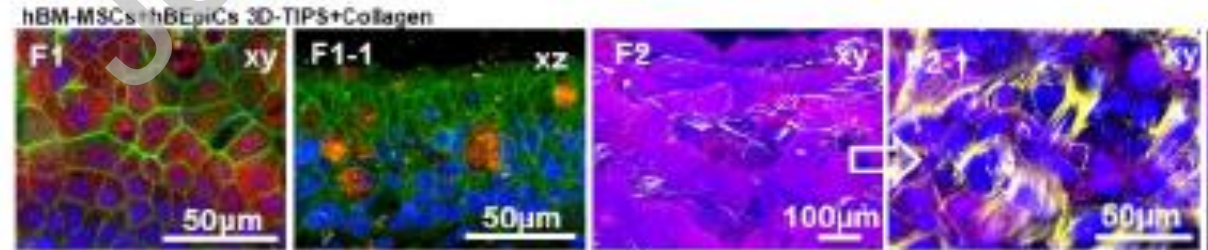

$50 y m$

- Nuclei $=$ E-cadherin = MUC5AC KRT14 = KRT 18

Fig. 6 (A1-A2) qPCR ( $n=5$ per scaffold type) and ELISA analysis ( $n=4$ per scaffold type) for MUC5AC as a marker protein of goblet cells of mucus production; (A3-A4) qPCR ( $n=5$ per scaffold type) and ELISA analysis ( $\mathrm{n}=4$ per scaffold type) for keratin 18 as a marker protein of differentiated cells. (B-F) 
Representative confocal microscope images ( $n=2$ per scaffold type) for the various scaffolds at day 21 showing (B1-F1) MUC5AC glycoprotein secretion, and (B2-F2) intracellular keratin 14 and intracellular keratin 18: (B) hBEpiCs monoculture on PET cell culture insert; (C) hBEpiCs monoculture on untreated 3D-TIPS; (D) hBEpiCs monoculture on 3D-TIPS+Collagen; (E) hBEpiCs co-culture with hBFs on 3DTIPS+Collagen; (F) hBEpiCs co-culture with hBM-MSCs on 3D-TIPS+Collagen. Maximum intensity projections of keratin 14 (yellow), keratin 18 (purple), and MUC5AC (red) reconstructed from z-stacks; cells counterstained for nuclei (blue) and e-catherin (green). Differences between the groups were analyzed by two-way ANOVA using Tukey's post hoc test. ${ }^{*} p<0.05 ;{ }^{* *} p<0.01 ;{ }^{* *} p<0.001$; $* * * * p<0.0001$. 

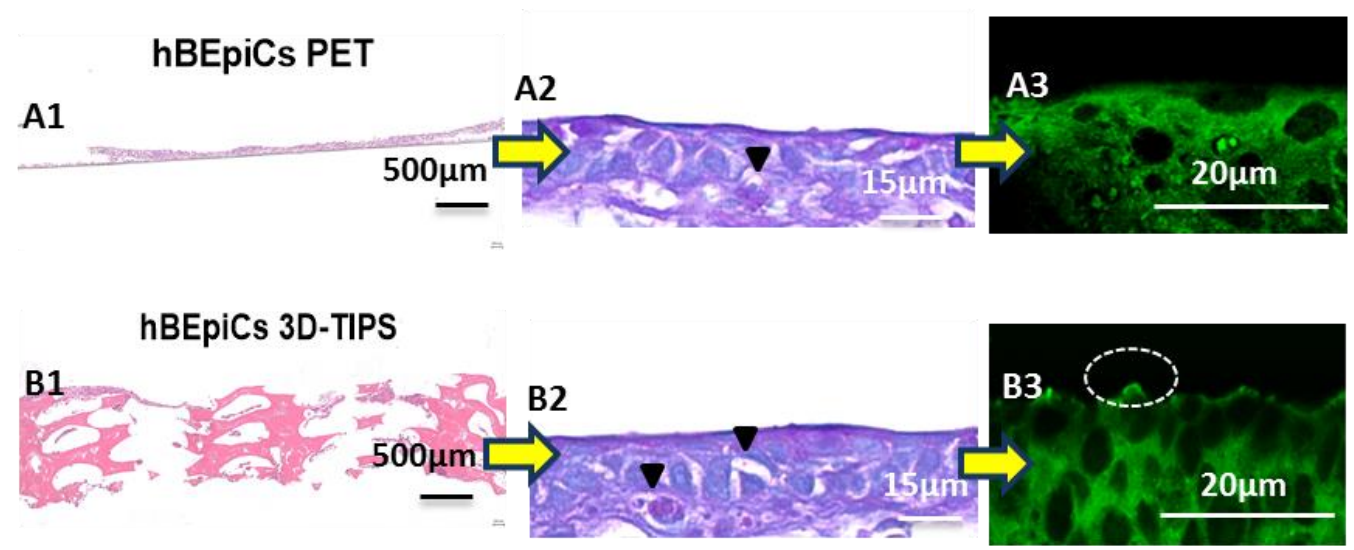

hBEpiCs 3D-TIPS+Collagen

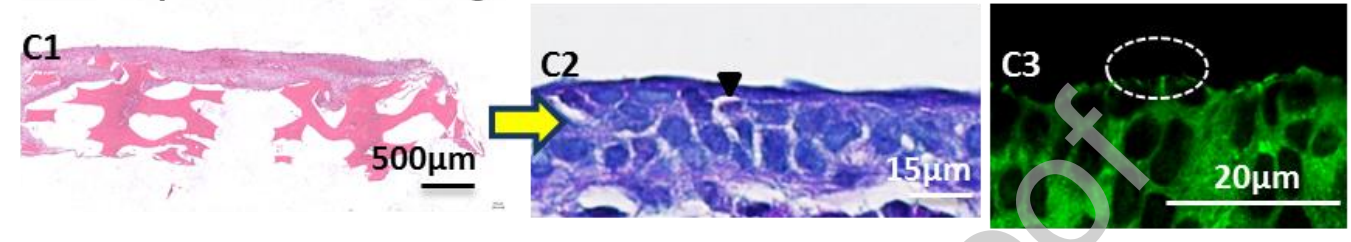

hBF+hBEpiCs 3D-TIPS+Collagen

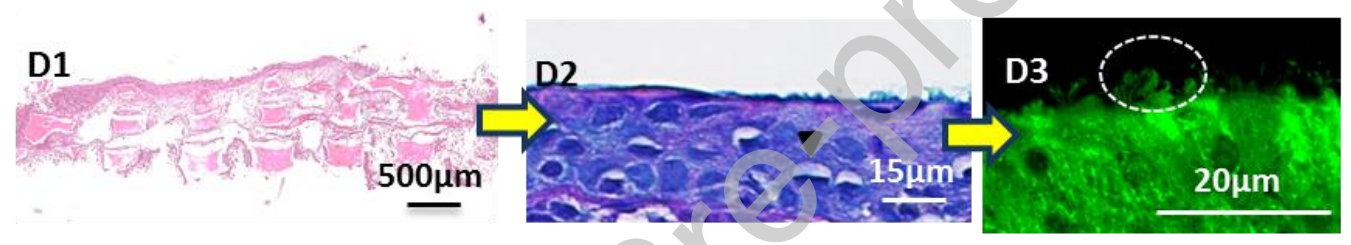

hBM-MSCs+hBEpiCs 3D-TIPS+Collagen

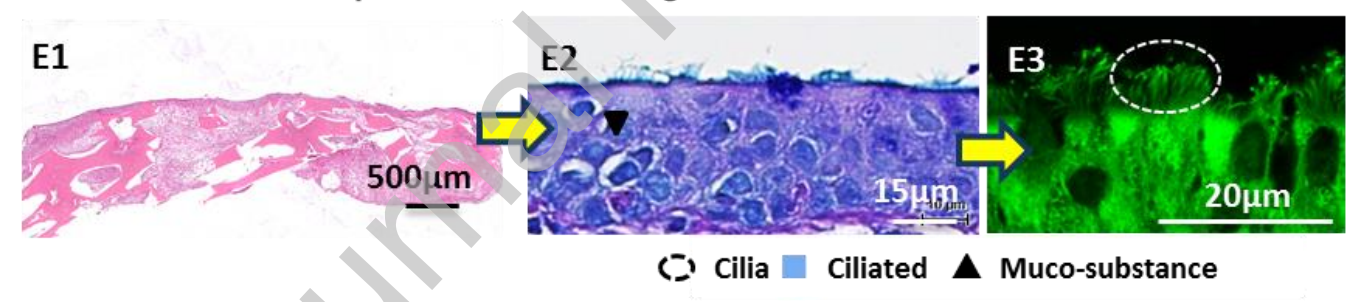

$\mathbf{F}$

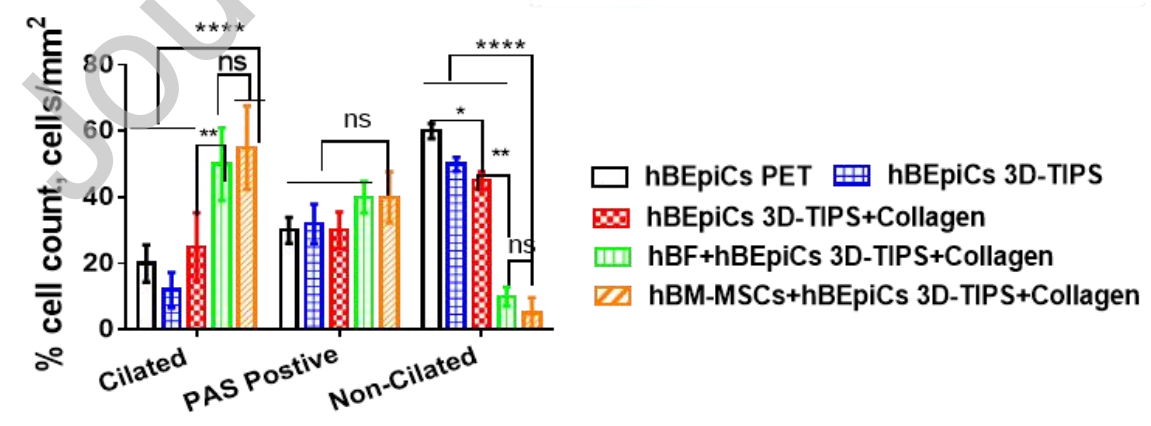

Fig. 7 Representative (A1-E1) Hematoxylin \& Eosin (H\&E), (A2-E2) Alcian blue-Periodic acid-Schiff (ABPAS), and (A3-F3) F-actin stained images at day 21 of cross-sections of differentiated hBEpiCs on the different scaffolds ( $n=2$ per type): (A) hBEpiCs monoculture on PET cell culture insert; (B) hBEpiCs 
monoculture on untreated 3D-TIPS; (C) hBEpiCs monoculture on 3D-TIPS+Collagen; (D) hBEpiCs cocultured with hBFs on 3D-TIPS+Collagen; (E) hBEpiCs co-cultured with hBM-MSCs on 3D-TIPS+Collagen. (F) Total percentage of cell count as seen by AB-PAS staining ( $n=2$ per scaffold type; 10 frames per scaffold with 500 cells analyzed). Differences between the groups were analyzed by two-way ANOVA using Tukey's post hoc test. n.s non-significant; ${ }^{*} \mathrm{p}<0.05 ;{ }^{* *} \mathrm{p}<0.01 ;{ }^{* * *} \mathrm{p}<0.0001$.

A

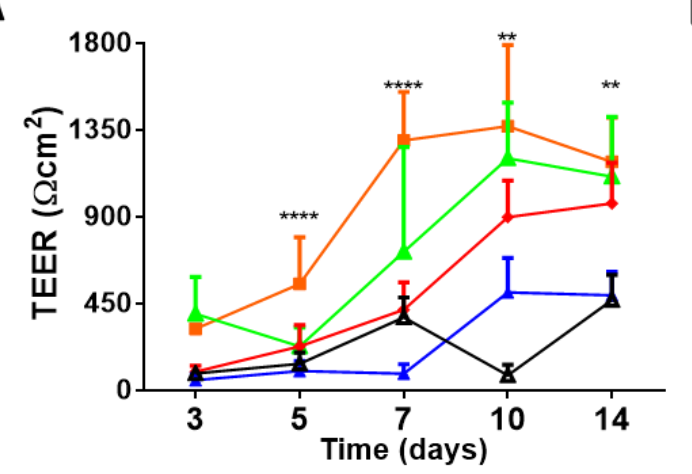

$\square$ hBEpiCs PET 㘞 hBEpiCs 3D-TIPS hBF+hBEpiCs 3D-TIPS+Collagen

C

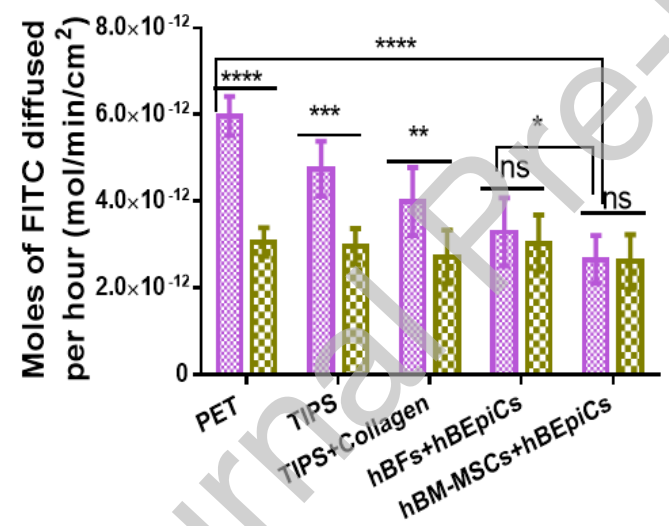

D

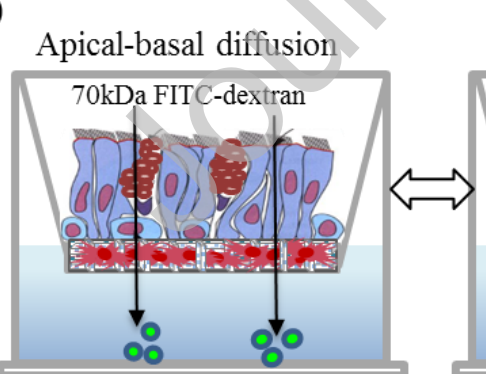
Basal-apical diffusion
B

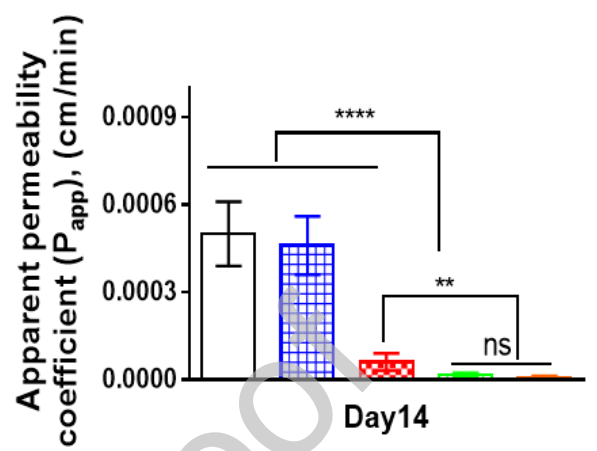

W hBEpiCs 3D-TIPS+Collagen

$\square$ hBM-MSCs+hBEpiCs 3D-TIPS+Collagen

Allergens, Viruses, Bacteria, Particulates
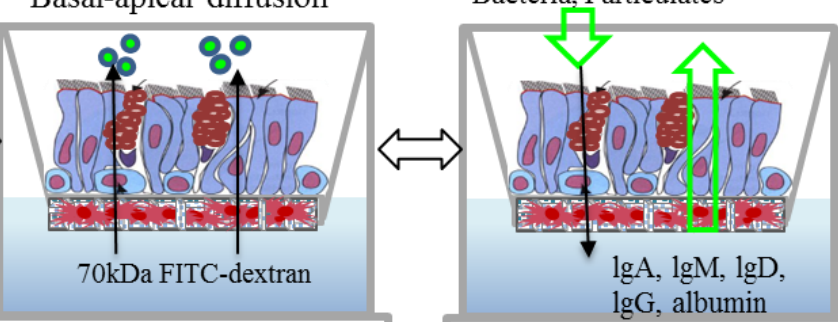

Fig. 8 Transepithelial electrical resistance (TEER) of hBEpiCs cultured in monoculture and co-culture conditions with hBM-MSCs or hBFs for 14 days at an air-liquid interface: (A) transepithelial electrical resistance (TEER) ( $n=6$ per scaffold type, 6 measurements per sample); (B) apparent permeability coefficient (Papp) of fluorescein isothiocyanate-labelled dextran 70 (FITC-dextran) through the bronchial 
epithelial cell barrier at day 14 ( $n=6$ per scaffold type, 6 measurements per sample); (C) molecular diffusion of FITC-dextran on day 21, basolateral to apical vs. apical to basolateral compartments ( $\mathrm{n}=6$ per scaffold type, 6 measurements per sample); (D) schematics showing apical to basolateral and basolateral to apical diffusion through the cell layer. Differences between the groups were analyzed by one-way or two-way ANOVA using Tukey's post hoc test. n.s non-significant; ${ }^{*} p<0.05 ;{ }^{* *} p<0.01$; $* * * p<0.001 ; * * * * p<0.0001$. 